\title{
The "staple" motif: a key to stability of thiolate-protected gold nanoclusters
}

\author{
De-en Jiang, ${ }^{,}{ }^{\dagger}$ Murilo L. Tiago, ${ }^{\ddagger}$ Weidong Luo, ${ }^{\ddagger, \S}$ and Sheng Dai ${ }^{\dagger}$ \\ ${ }^{\dagger}$ Chemical Sciences and ${ }^{\ddagger}$ Materials Science and Technology Divisions, Oak Ridge National \\ Laboratory, Oak Ridge, Tennessee 37831 and ${ }^{\S}$ Department of Physics and Astronomy, Vanderbilt \\ University, Nashville, Tennessee 37235 \\ E-mail: jiangd@ornl.gov
}

\section{Supporting Information}

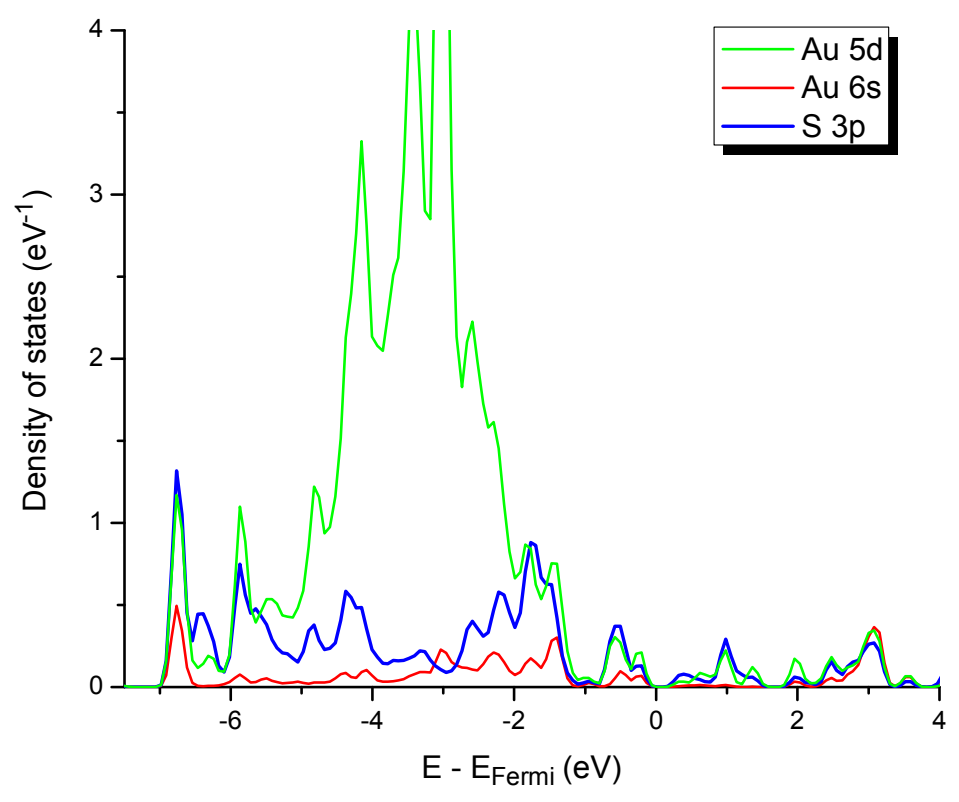

Figure S-1. Orbital-resolved local density of states for $\mathrm{S}$ and $\mathrm{Au}$ atoms in the "staple" motif of $\mathrm{Au}_{38}(\mathrm{MT})_{2}$ (see Figure 2d). 


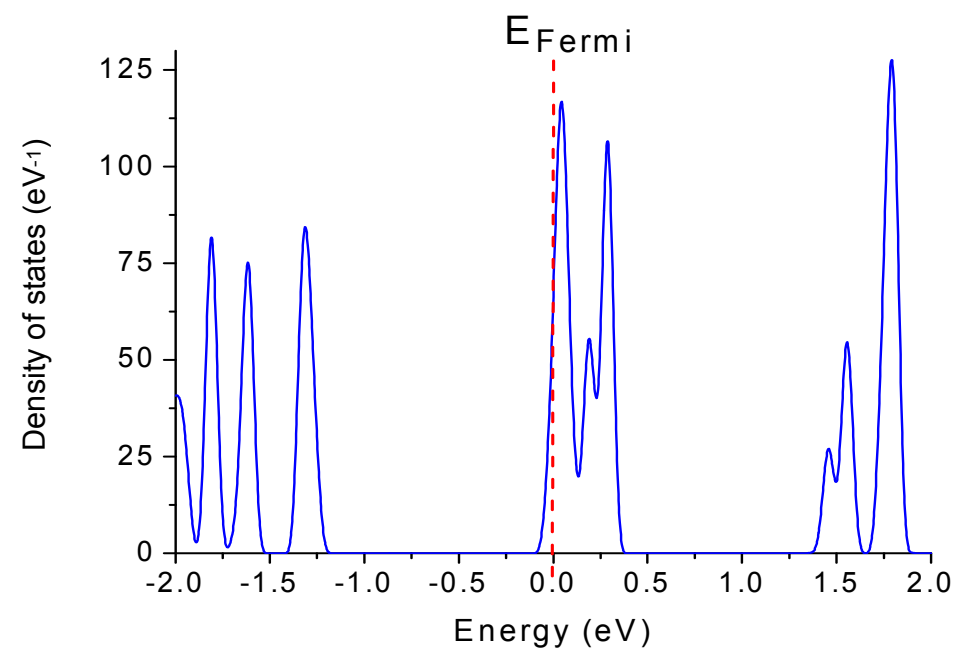

Figure S-2. Electronic density of states of $\mathrm{Au}_{38}$ in the $\mathrm{O}_{\mathrm{h}}$ symmetry (see Figure 2a).

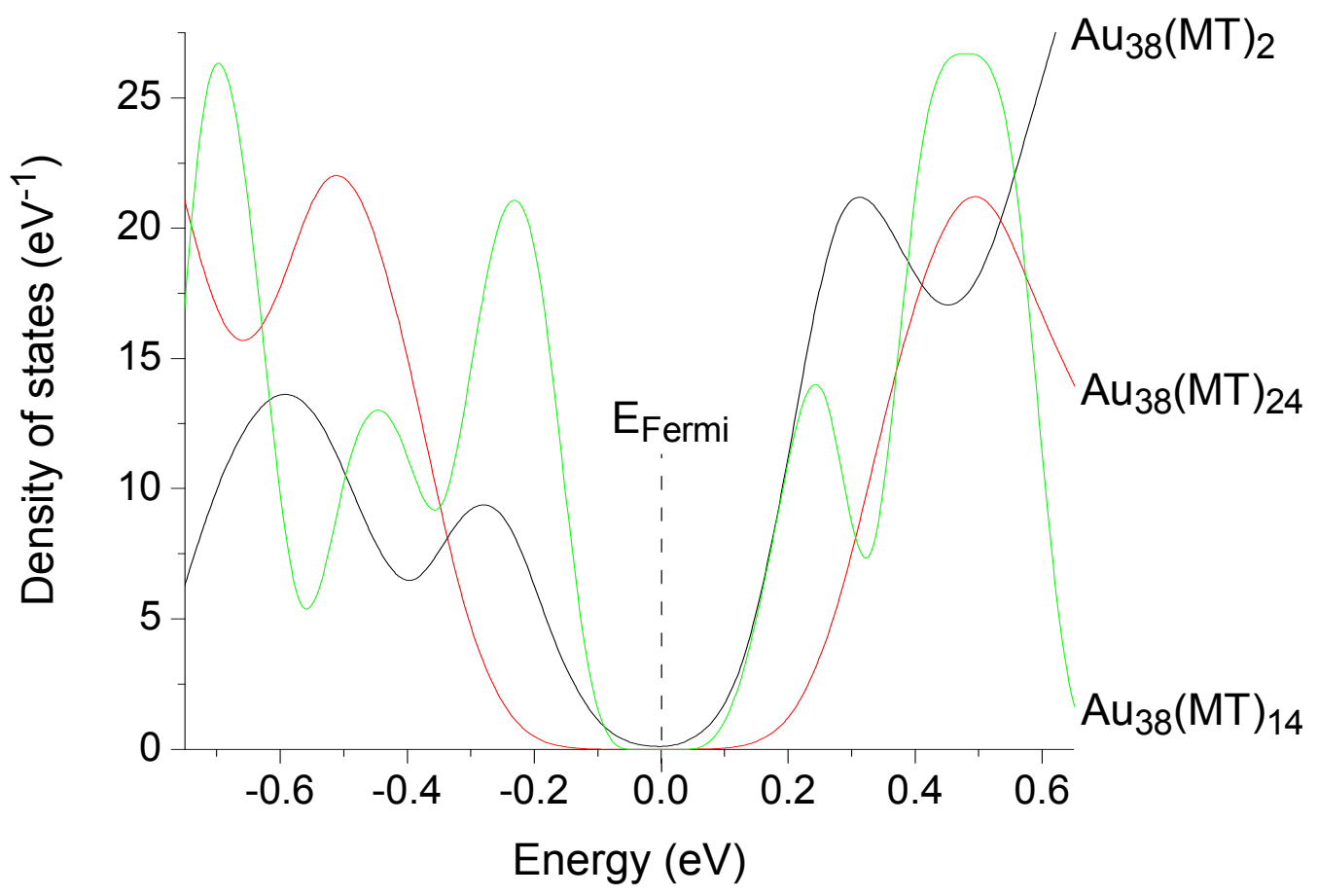

Figure S-3. Electronic density of states of $A_{38}(M T)_{2}$ (see Figure $2 d$ ), $A_{38}(M T)_{14}$ (see Figure 3), and $\mathrm{Au}_{38}(\mathrm{MT})_{24}$ (see Figure 5c). 


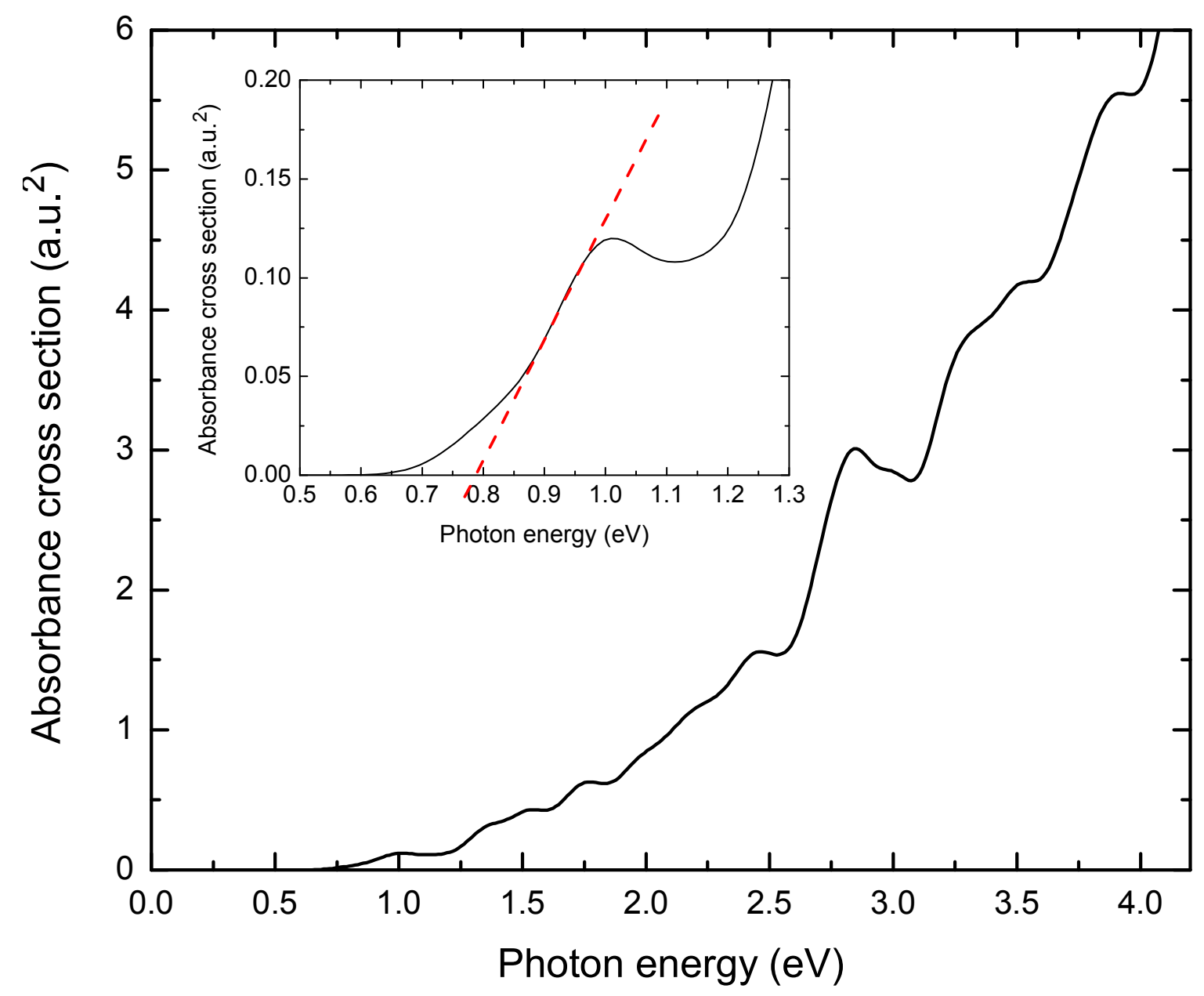

Figure S-4. Optical spectrum of $\mathrm{Au}_{38} \mathrm{MT}_{24}$ in the staple configuration (see Figure 5c), computed from time-dependent DFT. Inset is a zoom-in of the spectra from 0.5 to $1.3 \mathrm{eV}$ and shows how an extrapolated gap of $0.8 \mathrm{eV}$ is obtained (see Ref. 13 in the text for the experimental optical spectrum and gap energy).

Computation detail: Absorption spectra were obtained using time-dependent DFT in the local, adiabatic approximation (TD-LDA). The spectrum is evaluated as a first-order perturbation in the electron density produced by an external laser source. We calculated the energy of optical excitations by diagonalizing an eigenvalue problem for the density matrix, following the energy-representation, real-space formulation proposed by Casida. In order to simulate temperature and disorder effects, we applied a Gaussian convolution to the computed spectra, with dispersion $0.07 \mathrm{eV}$. More details can be found in Reference below.

M. E. Casida in "Recent Advances in Density-Functional Methods", Part I, edited by D. P. Chong, World Scientific, Singapore, 1995, p. 155. 
Cartesian coordinates for cluster structures in figures.

\begin{tabular}{|c|c|c|c|}
\hline \multicolumn{4}{|c|}{ \#figure 1c } \\
\hline$C$ & 21.585037 & 12.482000 & 21.506090 \\
\hline c & 10.455916 & 12.487240 & 7.720934 \\
\hline & 9.059876 & 18.077479 & 8.059270 \\
\hline & 22.971854 & 18.085894 & 21.028344 \\
\hline & 14.120302 & 18.442957 & 6.033161 \\
\hline & 17.888351 & 18.490123 & 23.097783 \\
\hline & 18.550106 & 17.190907 & 5.340894 \\
\hline & 13.514526 & 17.207342 & 23.863296 \\
\hline & 15.755846 & 12.087032 & 5.781039 \\
\hline & 16.302257 & 12.179055 & 23.432743 \\
\hline & 13.382638 & 8.371605 & 10.102243 \\
\hline & 18.548691 & 8.348354 & 19.099113 \\
\hline & 25.106493 & 15.007738 & 17.269392 \\
\hline & 6.913275 & 14.979189 & 11.962516 \\
\hline & 12.065374 & 23.427870 & 8.965448 \\
\hline & 19.854497 & 23.414996 & 20.180320 \\
\hline & 19.869224 & 21.568402 & 7.696622 \\
\hline 0 & 12.158370 & 21.590041 & 21.468811 \\
\hline & 20.933281 & 12.170001 & 7.264032 \\
\hline & 11.151147 & 12.179822 & 21.972029 \\
\hline & 24.137213 & 20.754325 & 17.440317 \\
\hline C & 7.928527 & 20.825400 & 11.755157 \\
\hline C & 22.358816 & 10.329626 & 16.436138 \\
\hline C & 9.555358 & 10.328720 & 12.684476 \\
\hline & 17.046216 & 23.858476 & 8.606187 \\
\hline 0 & 14.948715 & 23.961837 & 20.501071 \\
\hline 0 & 22.723087 & 17.051131 & 8.316096 \\
\hline $\mathrm{C}$ & 9.247619 & 16.971342 & 20.852577 \\
\hline C & 17.401588 & 8.908803 & 8.438331 \\
\hline C & 14.632823 & 8.888039 & 20.720281 \\
\hline C & 13.323035 & 7.041838 & 16.019308 \\
\hline C & 18.745634 & 7.094699 & 13.134329 \\
\hline C & 22.281318 & 9.243383 & 12.083144 \\
\hline C & 9.730942 & 9.291921 & 17.077990 \\
\hline C & 25.125663 & 17.636110 & 12.179698 \\
\hline $\mathrm{C}$ & 6.848835 & 17.638790 & 16.928999 \\
\hline C & 8.420557 & 21.811877 & 16.734705 \\
\hline $\mathrm{C}$ & 23.592974 & 21.775015 & 12.569909 \\
\hline$c$ & 18.829431 & 25.331587 & 12.412952 \\
\hline C & 13.084283 & 25.261497 & 16.670486 \\
\hline C & 24.898291 & 12.243286 & 11.521472 \\
\hline C & 7.039902 & 12.299749 & 17.626192 \\
\hline C & 12.620166 & 24.732947 & 13.182238 \\
\hline$c$ & 19.442718 & 24.725293 & 15.981764 \\
\hline $\mathrm{S}$ & 20.964854 & 14.133870 & 21.007773 \\
\hline $\mathrm{S}$ & 11.058168 & 14.151852 & 8.168896 \\
\hline $\mathrm{S}$ & 10.877745 & 18.216940 & 8.204457 \\
\hline $\mathrm{S}$ & 21.151165 & 18.222359 & 20.918763 \\
\hline $\mathrm{S}$ & 14.580230 & 19.792811 & 7.179827 \\
\hline S & 17.430331 & 19.867631 & 21.992202 \\
\hline S & 17.249582 & 16.235533 & 6.198487 \\
\hline S & 14.812202 & 16.302024 & 22.951176 \\
\hline $\mathrm{S}$ & 14.688496 & 12.953286 & 6.983336 \\
\hline $\mathrm{S}$ & 17.351133 & 12.986192 & 22.177966 \\
\hline $\mathrm{S}$ & 12.492703 & 9.934541 & 9.774977 \\
\hline $\mathrm{S}$ & 19.454960 & 9.906504 & 19.398230 \\
\hline $\mathrm{S}$ & 23.912824 & 16.160349 & 18.030647 \\
\hline $\mathrm{S}$ & 8.070282 & 16.141697 & 11.153934 \\
\hline $\mathrm{S}$ & 11.531167 & 22.312487 & 10.312185 \\
\hline $\mathrm{S}$ & 20.437647 & 22.312701 & 18.843874 \\
\hline $\mathrm{S}$ & 19.274744 & 19.851002 & 7.509773 \\
\hline $\mathrm{S}$ & 12.744488 & 19.872480 & 21.656302 \\
\hline $\mathrm{S}$ & 19.133500 & 12.197065 & 7.570091 \\
\hline $\mathrm{S}$ & 12.945269 & 12.240060 & 21.644783 \\
\hline $\mathrm{S}$ & 22.814587 & 20.520827 & 16.197835 \\
\hline S & 9.254090 & 20.548417 & 12.983039 \\
\hline $\mathrm{S}$ & 22.961781 & 12.049099 & 16.516193 \\
\hline
\end{tabular}




\begin{tabular}{|c|c|c|}
\hline 9.035989 & 12.080364 & 12.633122 \\
\hline 15.665473 & 22.829763 & 9.209762 \\
\hline 16.313822 & 22.891981 & 19.937701 \\
\hline 21.838984 & 15.500315 & 8.687459 \\
\hline 10.170643 & 15.449980 & 20.457547 \\
\hline 17.182878 & 9.330670 & 10.202609 \\
\hline 14.813552 & 9.301392 & 18.948511 \\
\hline 13.693331 & 8.174837 & 14.627478 \\
\hline 18.349593 & 8.204003 & 14.537542 \\
\hline 21.473414 & 10.738488 & 11.397729 \\
\hline 10.535115 & 10.780084 & 17.774332 \\
\hline 24.133176 & 16.922671 & 13.531956 \\
\hline 7.874216 & 16.941970 & 15.586689 \\
\hline 9.755994 & 20.989436 & 17.680017 \\
\hline 22.235173 & 21.023265 & 11.599846 \\
\hline 18.388718 & 23.584222 & 12.097642 \\
\hline 13.596667 & 23.548661 & 17.056984 \\
\hline 24.164066 & 13.708275 & 12.326015 \\
\hline 7.820012 & 13.735911 & 16.812042 \\
\hline 14.411728 & 24.412124 & 13.400919 \\
\hline 17.644084 & 24.438002 & 15.752132 \\
\hline 22.670969 & 12.441181 & 21.369476 \\
\hline 21.108620 & 11.681448 & 20.931372 \\
\hline 21.341398 & 12.360205 & 22.569307 \\
\hline 11.212949 & 12.022578 & 7.076194 \\
\hline 9.515684 & 12.605565 & 7.167430 \\
\hline 10.292524 & 11.860074 & 8.603367 \\
\hline 8.569688 & 18.089952 & 9.037917 \\
\hline 8.836489 & 17.129828 & 7.553091 \\
\hline 8.706953 & 18.920138 & 7.451863 \\
\hline 23.445089 & 18.104633 & 20.041570 \\
\hline 23.205879 & 17.135798 & 21.524636 \\
\hline 23.334722 & 18.925972 & 21.633695 \\
\hline 13.979582 & 18.889334 & 5.040326 \\
\hline 13.173355 & 18.011242 & 6.378707 \\
\hline 14.892338 & 17.667237 & 5.991957 \\
\hline 17.389560 & 17.559257 & 22.810025 \\
\hline 17.604710 & 18.758761 & 24.123519 \\
\hline 18.976755 & 18.365797 & 23.032705 \\
\hline 18.796884 & 16.662402 & 4.411536 \\
\hline 19.446224 & 17.300839 & 5.959457 \\
\hline 18.145430 & 18.184527 & 5.111629 \\
\hline 13.297088 & 16.644606 & 24.779725 \\
\hline 12.603306 & 17.318648 & 23.267188 \\
\hline 13.904055 & 18.201410 & 24.116290 \\
\hline 15.108757 & 11.465644 & 5.148829 \\
\hline 16.513791 & 11.467839 & 6.270331 \\
\hline 16.251137 & 12.853233 & 5.171156 \\
\hline 16.952088 & 11.562746 & 24.067310 \\
\hline 15.517427 & 11.561436 & 22.984655 \\
\hline 15.838358 & 12.972377 & 24.032560 \\
\hline 14.467514 & 8.521791 & 10.127728 \\
\hline 13.115008 & 7.660045 & 9.310455 \\
\hline 13.049422 & 7.985973 & 11.073615 \\
\hline 19.086423 & 7.799463 & 18.315868 \\
\hline 17.518833 & 8.536008 & 18.775088 \\
\hline 18.553270 & 7.764357 & 20.028338 \\
\hline 25.686143 & 15.570494 & 16.527634 \\
\hline 24.606072 & 14.163217 & 16.786745 \\
\hline 25.768151 & 14.637797 & 18.062642 \\
\hline 7.279968 & 13.948976 & 11.919598 \\
\hline 5.956677 & 15.052552 & 11.428895 \\
\hline 6.780638 & 15.286404 & 13.006998 \\
\hline 12.131578 & 24.440596 & 9.385088 \\
\hline 11.305514 & 23.404689 & 8.175312 \\
\hline 13.040991 & 23.139017 & 8.561085 \\
\hline 19.128425 & 22.914906 & 20.828869 \\
\hline 19.378788 & 24.288767 & 19.715813 \\
\hline 20.730155 & 23.729171 & 20.762523 \\
\hline 19.552554 & 22.153714 & 6.824417 \\
\hline 20.965373 & 21.528332 & 7.732736 \\
\hline
\end{tabular}




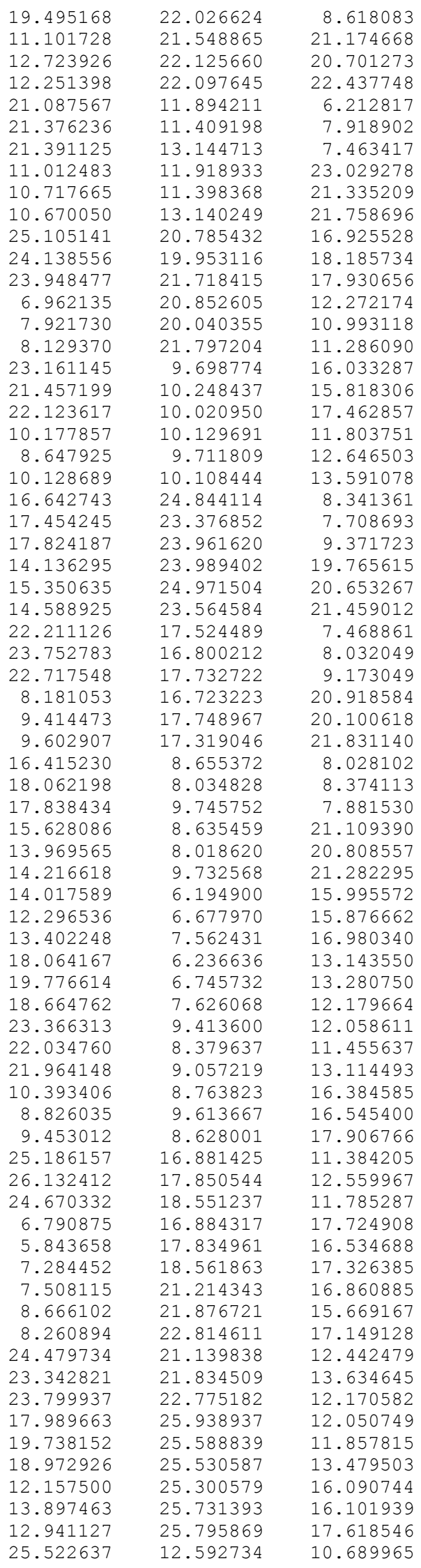




\begin{tabular}{|c|c|c|c|}
\hline $\mathrm{H}$ & 24.119071 & 11.570397 & 11.144266 \\
\hline $\mathrm{H}$ & 25.524083 & 11.727872 & 12.260817 \\
\hline $\mathrm{H}$ & 7.776366 & 11.522448 & 17.861309 \\
\hline $\mathrm{H}$ & 6.267756 & 11.903893 & 16.953781 \\
\hline $\mathrm{H}$ & 6.571984 & 12.651275 & 18.554094 \\
\hline $\mathrm{H}$ & 12.103128 & 24.495765 & 14.118440 \\
\hline $\mathrm{H}$ & 12.201019 & 24.114678 & 12.382081 \\
\hline $\mathrm{H}$ & 12.489954 & 25.797614 & 12.946729 \\
\hline $\mathrm{H}$ & 19.963245 & 24.502735 & 15.042187 \\
\hline $\mathrm{H}$ & 19.851452 & 24.080453 & 16.766969 \\
\hline $\mathrm{H}$ & 19.591315 & 25.780454 & 16.248172 \\
\hline $\mathrm{Au}$ & 14.642686 & 16.243694 & 10.192259 \\
\hline $\mathrm{Au}$ & 17.371645 & 16.267108 & 18.974496 \\
\hline $\mathrm{Au}$ & 16.459723 & 16.243301 & 16.025270 \\
\hline $\mathrm{Au}$ & 15.557020 & 16.234912 & 13.151126 \\
\hline $\mathrm{Au}$ & 17.038276 & 13.741791 & 17.464274 \\
\hline $\mathrm{Au}$ & 14.959601 & 13.743491 & 11.708525 \\
\hline $\mathrm{Au}$ & 19.216354 & 15.590489 & 16.790598 \\
\hline $\mathrm{Au}$ & 12.798184 & 15.601931 & 12.384080 \\
\hline $\mathrm{Au}$ & 18.225905 & 18.367597 & 17.087182 \\
\hline $\mathrm{Au}$ & 13.793386 & 18.351555 & 12.091527 \\
\hline $\mathrm{Au}$ & 15.457026 & 18.198540 & 17.974582 \\
\hline $\mathrm{Au}$ & 16.561080 & 18.168231 & 11.188725 \\
\hline $\mathrm{Au}$ & 17.293369 & 15.306498 & 10.933238 \\
\hline $\mathrm{Au}$ & 14.719158 & 15.321308 & 18.240542 \\
\hline $\mathrm{Au}$ & 16.003699 & 13.765742 & 14.590404 \\
\hline $\mathrm{Au}$ & 18.238107 & 15.476306 & 13.897152 \\
\hline $\mathrm{Au}$ & 13.771066 & 15.484636 & 15.270679 \\
\hline $\mathrm{Au}$ & 17.339242 & 18.261937 & 14.123358 \\
\hline $\mathrm{Au}$ & 14.664765 & 18.260184 & 15.034367 \\
\hline $\mathrm{Au}$ & 19.232343 & 14.057872 & 19.276602 \\
\hline $\mathrm{Au}$ & 12.772581 & 14.061127 & 9.896839 \\
\hline $\mathrm{Au}$ & 11.842756 & 17.132900 & 10.182768 \\
\hline $\mathrm{Au}$ & 20.168150 & 17.133748 & 18.967402 \\
\hline $\mathrm{Au}$ & 17.710073 & 19.024277 & 19.743218 \\
\hline $\mathrm{Au}$ & 14.285967 & 18.975823 & 9.426470 \\
\hline $\mathrm{Au}$ & 16.975427 & 16.938412 & 8.554008 \\
\hline $\mathrm{Au}$ & 15.035957 & 16.992438 & 20.593990 \\
\hline $\mathrm{Au}$ & 16.179197 & 13.964529 & 20.279758 \\
\hline $\mathrm{Au}$ & 15.859371 & 13.954739 & 8.872692 \\
\hline $\mathrm{Au}$ & 18.803214 & 12.903893 & 15.309736 \\
\hline $\mathrm{Au}$ & 13.206129 & 12.902811 & 13.870464 \\
\hline $\mathrm{Au}$ & 20.111621 & 17.526410 & 14.854999 \\
\hline $\mathrm{Au}$ & 11.870891 & 17.532915 & 14.324336 \\
\hline $\mathrm{Au}$ & 15.567558 & 20.518922 & 13.093460 \\
\hline $\mathrm{Au}$ & 16.432641 & 20.510619 & 16.069332 \\
\hline $\mathrm{Au}$ & 19.252009 & 17.420493 & 11.944857 \\
\hline $\mathrm{Au}$ & 12.755913 & 17.422456 & 17.235132 \\
\hline $\mathrm{Au}$ & 17.792207 & 12.898363 & 12.440550 \\
\hline $\mathrm{Au}$ & 14.204882 & 12.895326 & 16.734109 \\
\hline $\mathrm{Au}$ & 13.803000 & 11.338669 & 8.413692 \\
\hline $\mathrm{Au}$ & 18.167052 & 11.332734 & 20.751226 \\
\hline $\mathrm{Au}$ & 22.431948 & 14.949435 & 19.387279 \\
\hline $\mathrm{Au}$ & 9.553063 & 14.967813 & 9.760511 \\
\hline $\mathrm{Au}$ & 20.786765 & 20.246002 & 19.851579 \\
\hline $\mathrm{Au}$ & 11.228098 & 20.236763 & 9.294020 \\
\hline $\mathrm{Au}$ & 16.916471 & 19.837368 & 7.374468 \\
\hline $\mathrm{Au}$ & 15.095974 & 19.875485 & 21.809540 \\
\hline $\mathrm{Au}$ & 18.310778 & 14.300833 & 6.941562 \\
\hline $\mathrm{Au}$ & 13.748864 & 14.357371 & 22.235891 \\
\hline $\mathrm{Au}$ & 15.506690 & 11.240504 & 13.253795 \\
\hline $\mathrm{Au}$ & 16.487700 & 11.238038 & 15.903970 \\
\hline $\mathrm{Au}$ & 20.918566 & 14.679094 & 14.566585 \\
\hline $\mathrm{Au}$ & 11.085459 & 14.684152 & 14.612810 \\
\hline $\mathrm{Au}$ & 19.140381 & 20.260795 & 15.034526 \\
\hline $\mathrm{Au}$ & 12.847350 & 20.250342 & 14.127630 \\
\hline $\mathrm{Au}$ & 18.349233 & 20.225021 & 12.324683 \\
\hline $\mathrm{Au}$ & 13.652739 & 20.218418 & 16.826250 \\
\hline $\mathrm{Au}$ & 20.032247 & 14.624229 & 11.835089 \\
\hline $\mathrm{Au}$ & 11.983692 & 14.630807 & 17.334730 \\
\hline $\mathrm{Au}$ & 18.771162 & 11.492723 & 17.710624 \\
\hline
\end{tabular}




\begin{tabular}{|c|c|c|c|}
\hline $\mathrm{Au}$ & 13.224554 & 11.504580 & 11.456438 \\
\hline $\mathrm{Au}$ & 21.031063 & 13.391730 & 17.132066 \\
\hline $\mathrm{Au}$ & 10.987505 & 13.401770 & 12.055799 \\
\hline $\mathrm{Au}$ & 21.983832 & 16.453491 & 16.613027 \\
\hline $\mathrm{Au}$ & 10.036846 & 16.434664 & 12.525984 \\
\hline $\mathrm{Au}$ & 20.922071 & 19.246524 & 17.061045 \\
\hline $\mathrm{Au}$ & 11.093519 & 19.222505 & 12.083771 \\
\hline $\mathrm{Au}$ & 18.576772 & 21.175961 & 17.734587 \\
\hline $\mathrm{Au}$ & 13.406273 & 21.151461 & 11.416134 \\
\hline $\mathrm{Au}$ & 16.455672 & 20.827369 & 10.301407 \\
\hline $\mathrm{Au}$ & 15.534987 & 20.874935 & 18.859019 \\
\hline $\mathrm{Au}$ & 18.826807 & 18.991357 & 9.705242 \\
\hline $\mathrm{Au}$ & 13.182822 & 19.023997 & 19.457042 \\
\hline $\mathrm{Au}$ & 19.583129 & 16.027500 & 9.400813 \\
\hline $\mathrm{Au}$ & 12.432012 & 16.013201 & 19.783380 \\
\hline $\mathrm{Au}$ & 18.664117 & 13.023348 & 9.797080 \\
\hline $\mathrm{Au}$ & 13.362949 & 13.025977 & 19.387510 \\
\hline $\mathrm{Au}$ & 15.880607 & 11.477575 & 18.690453 \\
\hline $\mathrm{Au}$ & 16.111820 & 11.495834 & 10.460866 \\
\hline $\mathrm{Au}$ & 13.683470 & 10.472646 & 15.383407 \\
\hline $\mathrm{Au}$ & 18.314442 & 10.492624 & 13.779410 \\
\hline $\mathrm{Au}$ & 20.552146 & 12.145199 & 13.194897 \\
\hline $\mathrm{Au}$ & 11.452891 & 12.145178 & 15.964959 \\
\hline $\mathrm{Au}$ & 21.864232 & 16.657723 & 12.700695 \\
\hline $\mathrm{Au}$ & 10.132721 & 16.661973 & 16.467709 \\
\hline $\mathrm{Au}$ & 21.079466 & 19.429372 & 13.026401 \\
\hline $\mathrm{Au}$ & 10.912696 & 19.434681 & 16.179450 \\
\hline $\mathrm{Au}$ & 17.480320 & 22.432243 & 14.197840 \\
\hline $\mathrm{Au}$ & 14.513607 & 22.418010 & 14.968896 \\
\hline $\mathrm{Au}$ & 16.020355 & 8.365797 & 14.583199 \\
\hline $\mathrm{Au}$ & 23.220209 & 12.842453 & 14.315143 \\
\hline $\mathrm{Au}$ & 8.756583 & 12.852897 & 14.835468 \\
\hline $\mathrm{Au}$ & 20.345803 & 22.354955 & 11.937949 \\
\hline $\mathrm{Au}$ & 11.658963 & 22.305812 & 17.336598 \\
\hline $\mathrm{Au}$ & 12.719197 & 10.132751 & 18.314848 \\
\hline $\mathrm{Au}$ & 19.279513 & 10.127491 & 10.872471 \\
\hline $\mathrm{Au}$ & 23.423145 & 18.689645 & 14.879713 \\
\hline $\mathrm{Au}$ & 8.616022 & 18.721777 & 14.283229 \\
\hline $\mathrm{Au}$ & 16.945681 & 23.634680 & 17.810616 \\
\hline $\mathrm{Au}$ & 15.081964 & 23.588685 & 11.342655 \\
\hline $\mathrm{Au}$ & 22.774010 & 14.649071 & 10.666764 \\
\hline $\mathrm{Au}$ & 9.236372 & 14.629499 & 18.470097 \\
\hline \multicolumn{4}{|c|}{ \#figure $2 a$} \\
\hline $\mathrm{Au}$ & 12.178855 & 12.061600 & 12.950000 \\
\hline $\mathrm{Au}$ & 8.195570 & 12.061600 & 12.950000 \\
\hline $\mathrm{Au}$ & 10.187225 & 14.053230 & 12.950000 \\
\hline $\mathrm{Au}$ & 10.187225 & 10.069970 & 12.950000 \\
\hline $\mathrm{Au}$ & 10.187225 & 12.061600 & 14.941655 \\
\hline $\mathrm{Au}$ & 10.187225 & 12.061600 & 10.958370 \\
\hline $\mathrm{Au}$ & 12.389592 & 14.263967 & 15.152367 \\
\hline $\mathrm{Au}$ & 7.984858 & 14.263967 & 15.152367 \\
\hline $\mathrm{Au}$ & 12.389592 & 9.859233 & 15.152367 \\
\hline $\mathrm{Au}$ & 12.389592 & 14.263967 & 10.747633 \\
\hline $\mathrm{Au}$ & 12.389592 & 9.859233 & 10.747633 \\
\hline $\mathrm{Au}$ & 7.984858 & 14.263967 & 10.747633 \\
\hline $\mathrm{Au}$ & 7.984858 & 9.859233 & 15.152367 \\
\hline $\mathrm{Au}$ & 7.984858 & 9.859233 & 10.747633 \\
\hline $\mathrm{Au}$ & 12.196643 & 16.061682 & 12.950000 \\
\hline $\mathrm{Au}$ & 12.196643 & 8.061518 & 12.950000 \\
\hline $\mathrm{Au}$ & 12.196643 & 12.061600 & 16.950082 \\
\hline $\mathrm{Au}$ & 12.196643 & 12.061600 & 8.949918 \\
\hline $\mathrm{Au}$ & 8.177782 & 16.061682 & 12.950000 \\
\hline $\mathrm{Au}$ & 8.177782 & 8.061518 & 12.950000 \\
\hline $\mathrm{Au}$ & 8.177782 & 12.061600 & 16.950082 \\
\hline $\mathrm{Au}$ & 8.177782 & 12.061600 & 8.949918 \\
\hline $\mathrm{Au}$ & 14.187307 & 14.071018 & 12.950000 \\
\hline $\mathrm{Au}$ & 6.187143 & 14.071018 & 12.950000 \\
\hline $\mathrm{Au}$ & 10.187225 & 14.071018 & 16.950082 \\
\hline $\mathrm{Au}$ & 10.187225 & 14.071018 & 8.949918 \\
\hline $\mathrm{Au}$ & 14.187307 & 10.052182 & 12.950000 \\
\hline
\end{tabular}




$\begin{array}{lrrr}\mathrm{Au} & 6.187143 & 10.052182 & 12.950000 \\ \mathrm{Au} & 10.187225 & 10.052182 & 16.950082 \\ \mathrm{Au} & 10.187225 & 10.052182 & 8.949918 \\ \mathrm{Au} & 14.187307 & 12.061600 & 14.959418 \\ \mathrm{Au} & 6.187143 & 12.061600 & 14.959418 \\ \mathrm{Au} & 10.187225 & 16.061682 & 14.959418 \\ \mathrm{Au} & 10.187225 & 8.061518 & 14.959418 \\ \mathrm{Au} & 14.187307 & 12.061600 & 10.940582 \\ \mathrm{Au} & 6.187143 & 12.061600 & 10.940582 \\ \mathrm{Au} & 10.187225 & 16.061682 & 10.940582 \\ \mathrm{Au} & 10.187225 & 8.061518 & 10.940582\end{array}$

\begin{tabular}{|c|c|c|c|}
\hline \multicolumn{4}{|c|}{ \#figure 2b } \\
\hline C & 7.760025 & 5.643136 & 15.575076 \\
\hline S & 8.897422 & 6.142864 & 14.243226 \\
\hline $\mathrm{H}$ & 7.563725 & 6.467422 & 16.271840 \\
\hline $\mathrm{H}$ & 8.222232 & 4.800696 & 16.107351 \\
\hline $\mathrm{H}$ & 6.814461 & 5.308418 & 15.117170 \\
\hline $\mathrm{Au}$ & 12.146070 & 12.035804 & 12.942862 \\
\hline $\mathrm{Au}$ & 8.189986 & 12.047363 & 12.942243 \\
\hline $\mathrm{Au}$ & 10.219217 & 14.040732 & 12.919871 \\
\hline $\mathrm{Au}$ & 10.082469 & 9.996110 & 13.066011 \\
\hline $\mathrm{Au}$ & 10.194144 & 12.051017 & 14.944265 \\
\hline $\mathrm{Au}$ & 10.200078 & 12.038207 & 10.993761 \\
\hline $\mathrm{Au}$ & 12.449466 & 14.332081 & 15.179910 \\
\hline $\mathrm{Au}$ & 7.977368 & 14.310139 & 15.167986 \\
\hline $\mathrm{Au}$ & 12.391848 & 9.783445 & 15.233145 \\
\hline $\mathrm{Au}$ & 12.489926 & 14.385597 & 10.645772 \\
\hline $\mathrm{Au}$ & 12.415273 & 9.868629 & 10.715848 \\
\hline $\mathrm{Au}$ & 7.974801 & 14.322061 & 10.703565 \\
\hline $\mathrm{Au}$ & 8.055307 & 9.930744 & 15.076934 \\
\hline $\mathrm{Au}$ & 7.900327 & 9.794151 & 10.787983 \\
\hline $\mathrm{Au}$ & 12.197863 & 16.094280 & 12.894454 \\
\hline $\mathrm{Au}$ & 12.187616 & 8.126777 & 12.937910 \\
\hline $\mathrm{Au}$ & 12.182025 & 12.056126 & 16.987298 \\
\hline $\mathrm{Au}$ & 12.184890 & 12.112511 & 8.958778 \\
\hline $\mathrm{Au}$ & 8.227452 & 16.059441 & 12.919699 \\
\hline $\mathrm{Au}$ & 7.968261 & 7.914778 & 12.881483 \\
\hline $\mathrm{Au}$ & 8.210542 & 12.088484 & 16.937007 \\
\hline $\mathrm{Au}$ & 8.176430 & 12.058605 & 9.054655 \\
\hline $\mathrm{Au}$ & 14.146944 & 14.087611 & 12.959302 \\
\hline $\mathrm{Au}$ & 6.237175 & 14.081844 & 12.930013 \\
\hline $\mathrm{Au}$ & 10.218395 & 14.080853 & 16.908571 \\
\hline $\mathrm{Au}$ & 10.181861 & 14.088854 & 8.981768 \\
\hline $\mathrm{Au}$ & 14.165491 & 10.084704 & 12.964956 \\
\hline $\mathrm{Au}$ & 6.139733 & 10.028723 & 12.950961 \\
\hline $\mathrm{Au}$ & 10.199859 & 10.001557 & 16.952429 \\
\hline $\mathrm{Au}$ & 10.169443 & 10.085920 & 8.981791 \\
\hline $\mathrm{Au}$ & 14.096395 & 12.060411 & 14.954722 \\
\hline $\mathrm{Au}$ & 6.190248 & 12.098152 & 14.942161 \\
\hline $\mathrm{Au}$ & 10.228272 & 16.057323 & 14.918893 \\
\hline $\mathrm{Au}$ & 10.350104 & 7.877375 & 15.126978 \\
\hline $\mathrm{Au}$ & 14.160400 & 12.106979 & 10.946650 \\
\hline $\mathrm{Au}$ & 6.132082 & 12.057322 & 10.960672 \\
\hline $\mathrm{Au}$ & 10.249022 & 16.085845 & 10.939540 \\
\hline $\mathrm{Au}$ & 10.173175 & 8.135160 & 10.983641 \\
\hline \multicolumn{4}{|c|}{ \#figure 2c } \\
\hline S & 8.750700 & 6.514850 & 14.359400 \\
\hline S & 6.818925 & 8.505650 & 11.476800 \\
\hline C & 7.768125 & 5.632325 & 15.592900 \\
\hline C & 6.840200 & 7.252250 & 10.175150 \\
\hline $\mathrm{H}$ & 7.555525 & 6.323125 & 16.474500 \\
\hline $\mathrm{H}$ & 8.345700 & 4.719150 & 15.956350 \\
\hline $\mathrm{H}$ & 6.783350 & 5.298000 & 15.125975 \\
\hline $\mathrm{H}$ & 7.817150 & 6.667025 & 10.227075 \\
\hline $\mathrm{H}$ & 6.754700 & 7.759475 & 9.157775 \\
\hline $\mathrm{H}$ & 5.962200 & 6.539600 & 10.319425 \\
\hline $\mathrm{Au}$ & 12.178425 & 12.061600 & 12.950000 \\
\hline $\mathrm{Au}$ & 8.196000 & 12.061600 & 12.950000 \\
\hline
\end{tabular}




\begin{tabular}{|c|c|c|c|}
\hline $\mathrm{Au}$ & 10.187225 & 14.052800 & 12.950000 \\
\hline $\mathrm{Au}$ & 10.187225 & 10.070400 & 12.950000 \\
\hline $\mathrm{Au}$ & 10.187225 & 12.061600 & 14.941225 \\
\hline $\mathrm{Au}$ & 10.187225 & 12.061600 & 10.958800 \\
\hline $\mathrm{Au}$ & 12.371875 & 14.246250 & 15.134650 \\
\hline $\mathrm{Au}$ & 8.002575 & 14.246250 & 15.134650 \\
\hline $\mathrm{Au}$ & 12.371875 & 9.876950 & 15.134650 \\
\hline $\mathrm{Au}$ & 12.371875 & 14.246250 & 10.765350 \\
\hline $\mathrm{Au}$ & 12.371875 & 9.876950 & 10.765350 \\
\hline $\mathrm{Au}$ & 8.002575 & 14.246250 & 10.765350 \\
\hline $\mathrm{Au}$ & 8.002575 & 9.876950 & 15.134650 \\
\hline $\mathrm{Au}$ & 8.002575 & 9.876950 & 10.765350 \\
\hline $\mathrm{Au}$ & 12.201125 & 16.061400 & 12.950000 \\
\hline $\mathrm{Au}$ & 12.201125 & 8.061800 & 12.950000 \\
\hline $\mathrm{Au}$ & 12.201125 & 12.061600 & 16.949800 \\
\hline $\mathrm{Au}$ & 12.201125 & 12.061600 & 8.950200 \\
\hline $\mathrm{Au}$ & 8.173300 & 16.061400 & 12.950000 \\
\hline $\mathrm{Au}$ & 8.173300 & 8.061800 & 12.950000 \\
\hline $\mathrm{Au}$ & 8.173300 & 12.061600 & 16.949800 \\
\hline $\mathrm{Au}$ & 8.173300 & 12.061600 & 8.950200 \\
\hline $\mathrm{Au}$ & 14.187025 & 14.075500 & 12.950000 \\
\hline $\mathrm{Au}$ & 6.187425 & 14.075500 & 12.950000 \\
\hline $\mathrm{Au}$ & 10.187225 & 14.075500 & 16.949800 \\
\hline $\mathrm{Au}$ & 10.187225 & 14.075500 & 8.950200 \\
\hline $\mathrm{Au}$ & 14.187025 & 10.047700 & 12.950000 \\
\hline $\mathrm{Au}$ & 6.187425 & 10.047700 & 12.950000 \\
\hline $\mathrm{Au}$ & 10.187225 & 10.047700 & 16.949800 \\
\hline $\mathrm{Au}$ & 10.187225 & 10.047700 & 8.950200 \\
\hline $\mathrm{Au}$ & 14.187025 & 12.061600 & 14.963900 \\
\hline $\mathrm{Au}$ & 6.187425 & 12.061600 & 14.963900 \\
\hline $\mathrm{Au}$ & 10.187225 & 16.061400 & 14.963900 \\
\hline $\mathrm{Au}$ & 10.187225 & 8.061800 & 14.963900 \\
\hline $\mathrm{Au}$ & 14.187025 & 12.061600 & 10.936100 \\
\hline $\mathrm{Au}$ & 6.187425 & 12.061600 & 10.936100 \\
\hline $\mathrm{Au}$ & 10.187225 & 16.061400 & 10.936100 \\
\hline $\mathrm{Au}$ & 10.187225 & 8.061800 & 10.936100 \\
\hline \multicolumn{4}{|c|}{ \#figure 2d } \\
\hline $\mathrm{S}$ & 9.455949 & 5.705472 & 14.253807 \\
\hline $\mathrm{S}$ & 6.400824 & 7.345406 & 11.080287 \\
\hline C & 8.291631 & 5.425430 & 15.635216 \\
\hline C & 6.534921 & 6.384975 & 9.533909 \\
\hline $\mathrm{H}$ & 7.762980 & 6.343775 & 15.909745 \\
\hline $\mathrm{H}$ & 8.867169 & 5.053299 & 16.491431 \\
\hline $\mathrm{H}$ & 7.570393 & 4.661856 & 15.318632 \\
\hline $\mathrm{H}$ & 7.559431 & 6.366968 & 9.149856 \\
\hline $\mathrm{H}$ & 5.872808 & 6.862692 & 8.800613 \\
\hline $\mathrm{H}$ & 6.184704 & 5.364652 & 9.731188 \\
\hline $\mathrm{Au}$ & 11.854962 & 12.460662 & 12.176880 \\
\hline $\mathrm{Au}$ & 6.560408 & 11.186713 & 13.042790 \\
\hline $\mathrm{Au}$ & 9.688736 & 13.962314 & 12.870628 \\
\hline $\mathrm{Au}$ & 8.622538 & 9.324240 & 13.700711 \\
\hline $\mathrm{Au}$ & 10.301963 & 11.715957 & 14.305915 \\
\hline $\mathrm{Au}$ & 9.253404 & 11.414501 & 11.789646 \\
\hline $\mathrm{Au}$ & 12.800230 & 14.538368 & 15.419495 \\
\hline $\mathrm{Au}$ & 8.143704 & 14.927918 & 15.177635 \\
\hline $\mathrm{Au}$ & 12.369506 & 9.859005 & 15.263704 \\
\hline $\mathrm{Au}$ & 13.007909 & 14.703580 & 10.751884 \\
\hline $\mathrm{Au}$ & 12.511145 & 10.025271 & 10.628399 \\
\hline $\mathrm{Au}$ & 8.288882 & 14.915844 & 10.480053 \\
\hline $\mathrm{Au}$ & 7.165006 & 10.397654 & 15.800267 \\
\hline $\mathrm{Au}$ & 7.517893 & 9.473334 & 10.892817 \\
\hline $\mathrm{Au}$ & 13.041483 & 16.082464 & 13.162700 \\
\hline $\mathrm{Au}$ & 12.567916 & 8.465213 & 12.871508 \\
\hline $\mathrm{Au}$ & 11.600995 & 12.220815 & 16.764959 \\
\hline $\mathrm{Au}$ & 11.895498 & 12.407251 & 9.208676 \\
\hline $\mathrm{Au}$ & 8.309047 & 16.464604 & 12.837695 \\
\hline $\mathrm{Au}$ & 8.055138 & 6.609888 & 12.589114 \\
\hline $\mathrm{Au}$ & 8.741907 & 12.563151 & 16.621850 \\
\hline $\mathrm{Au}$ & 9.127216 & 12.779417 & 8.923385 \\
\hline $\mathrm{Au}$ & 14.299682 & 13.628343 & 13.130596 \\
\hline
\end{tabular}




\begin{tabular}{|c|c|c|c|}
\hline $\mathrm{Au}$ & 6.797146 & 14.073426 & 12.741645 \\
\hline $\mathrm{Au}$ & 10.412307 & 14.743083 & 16.768533 \\
\hline $\mathrm{Au}$ & 10.777728 & 14.974334 & 9.156050 \\
\hline $\mathrm{Au}$ & 14.096341 & 10.714338 & 13.041167 \\
\hline $\mathrm{Au}$ & 4.602982 & 10.817766 & 15.007040 \\
\hline $\mathrm{Au}$ & 9.806199 & 9.946450 & 16.347173 \\
\hline $\mathrm{Au}$ & 10.030747 & 10.244248 & 9.364228 \\
\hline $\mathrm{Au}$ & 14.017585 & 12.083146 & 15.422761 \\
\hline $\mathrm{Au}$ & 6.247188 & 12.995107 & 15.227538 \\
\hline $\mathrm{Au}$ & 10.622001 & 16.202942 & 14.447926 \\
\hline $\mathrm{Au}$ & 10.667723 & 7.711049 & 14.794939 \\
\hline $\mathrm{Au}$ & 14.269864 & 12.237031 & 10.700531 \\
\hline $\mathrm{Au}$ & 6.957670 & 12.338596 & 10.540727 \\
\hline $\mathrm{Au}$ & 10.791344 & 16.307787 & 11.563312 \\
\hline $\mathrm{Au}$ & 10.188195 & 8.521917 & 11.525814 \\
\hline \multicolumn{4}{|c|}{ \#figure $3 a$} \\
\hline $\mathrm{S}$ & 9.385262 & 5.830044 & 14.202980 \\
\hline $\mathrm{S}$ & 6.323198 & 7.443391 & 11.035407 \\
\hline S & 5.028175 & 9.145811 & 16.710347 \\
\hline $\mathrm{S}$ & 3.521077 & 11.455430 & 12.952896 \\
\hline C & 8.305087 & 5.444862 & 15.629687 \\
\hline $\mathrm{C}$ & 6.777643 & 6.467210 & 9.552757 \\
\hline $\mathrm{C}$ & 4.020777 & 9.736638 & 18.121109 \\
\hline C & 2.696860 & 10.200997 & 11.900382 \\
\hline $\mathrm{H}$ & 7.706812 & 6.310897 & 15.929931 \\
\hline $\mathrm{H}$ & 8.946881 & 5.128161 & 16.460767 \\
\hline $\mathrm{H}$ & 7.644401 & 4.618486 & 15.338910 \\
\hline $\mathrm{H}$ & 7.840290 & 6.567200 & 9.311357 \\
\hline $\mathrm{H}$ & 6.169850 & 6.824121 & 8.712175 \\
\hline $\mathrm{H}$ & 6.539730 & 5.415337 & 9.756573 \\
\hline $\mathrm{H}$ & 4.029470 & 10.828172 & 18.195464 \\
\hline $\mathrm{H}$ & 4.434617 & 9.294520 & 19.035598 \\
\hline $\mathrm{H}$ & 2.994408 & 9.381081 & 17.970177 \\
\hline $\mathrm{H}$ & 3.384654 & 9.402866 & 11.603353 \\
\hline $\mathrm{H}$ & 2.327359 & 10.726397 & 11.010756 \\
\hline $\mathrm{H}$ & 1.850080 & 9.781077 & 12.456166 \\
\hline $\mathrm{Au}$ & 11.760459 & 12.190607 & 12.118198 \\
\hline $\mathrm{Au}$ & 5.720087 & 11.680855 & 12.077488 \\
\hline $\mathrm{Au}$ & 10.016783 & 14.094353 & 12.827121 \\
\hline $\mathrm{Au}$ & 8.255399 & 9.546782 & 14.052338 \\
\hline $\mathrm{Au}$ & 10.347983 & 11.880230 & 14.432481 \\
\hline $\mathrm{Au}$ & 8.738140 & 11.617806 & 12.241503 \\
\hline $\mathrm{Au}$ & 12.835387 & 14.483088 & 15.499700 \\
\hline $\mathrm{Au}$ & 8.211329 & 14.794047 & 15.028746 \\
\hline $\mathrm{Au}$ & 12.333494 & 9.875572 & 15.250924 \\
\hline $\mathrm{Au}$ & 13.155417 & 14.572742 & 10.864353 \\
\hline $\mathrm{Au}$ & 12.326117 & 9.948597 & 10.547065 \\
\hline $\mathrm{Au}$ & 8.648955 & 15.093829 & 10.325867 \\
\hline $\mathrm{Au}$ & 7.076012 & 10.340477 & 16.621425 \\
\hline $\mathrm{Au}$ & 7.399742 & 9.583156 & 10.926734 \\
\hline $\mathrm{Au}$ & 13.184577 & 15.966663 & 13.239158 \\
\hline $\mathrm{Au}$ & 12.553754 & 8.428819 & 12.846255 \\
\hline $\mathrm{Au}$ & 11.653618 & 12.254764 & 16.874406 \\
\hline $\mathrm{Au}$ & 11.980269 & 12.446461 & 9.190899 \\
\hline $\mathrm{Au}$ & 8.513313 & 16.505268 & 12.799227 \\
\hline $\mathrm{Au}$ & 7.921123 & 6.794410 & 12.643601 \\
\hline $\mathrm{Au}$ & 8.739342 & 12.578529 & 16.771589 \\
\hline $\mathrm{Au}$ & 9.427011 & 13.177862 & 8.377965 \\
\hline $\mathrm{Au}$ & 14.308776 & 13.467107 & 13.198085 \\
\hline $\mathrm{Au}$ & 7.104017 & 14.117950 & 12.462181 \\
\hline $\mathrm{Au}$ & 10.396973 & 14.752348 & 16.752438 \\
\hline $\mathrm{Au}$ & 11.153135 & 15.166592 & 9.102431 \\
\hline $\mathrm{Au}$ & 14.091254 & 10.657009 & 13.143083 \\
\hline $\mathrm{Au}$ & 4.320660 & 10.335829 & 14.829458 \\
\hline $\mathrm{Au}$ & 9.830965 & 10.022088 & 16.426788 \\
\hline $\mathrm{Au}$ & 9.750699 & 10.763232 & 9.714890 \\
\hline $\mathrm{Au}$ & 14.069989 & 12.011958 & 15.551593 \\
\hline $\mathrm{Au}$ & 7.084527 & 12.222150 & 14.547778 \\
\hline $\mathrm{Au}$ & 10.760009 & 16.279604 & 14.499778 \\
\hline $\mathrm{Au}$ & 10.521236 & 7.872375 & 14.726238 \\
\hline
\end{tabular}




\begin{tabular}{|c|c|c|c|}
\hline $\mathrm{Au}$ & 14.256824 & 12.031505 & 10.742893 \\
\hline $\mathrm{Au}$ & 7.395482 & 12.521351 & 10.048887 \\
\hline $\mathrm{Au}$ & 11.027213 & 16.413770 & 11.561911 \\
\hline $\mathrm{Au}$ & 10.024498 & 8.999519 & 11.914835 \\
\hline \multicolumn{4}{|c|}{ \#figure 3b } \\
\hline S & 9.315853 & 6.026756 & 13.951079 \\
\hline $\mathrm{S}$ & 6.250376 & 7.682640 & 10.815768 \\
\hline $\mathrm{S}$ & 4.852400 & 9.054177 & 16.873537 \\
\hline S & 3.266219 & 11.277707 & 13.081666 \\
\hline $\mathrm{S}$ & 14.223391 & 8.005117 & 15.459822 \\
\hline $\mathrm{S}$ & 13.289858 & 7.677109 & 10.893230 \\
\hline S & 13.899513 & 16.513285 & 15.307662 \\
\hline $\mathrm{S}$ & 14.821479 & 16.091885 & 10.739122 \\
\hline S & 7.836969 & 15.698599 & 7.857605 \\
\hline S & 8.795006 & 11.183481 & 7.142874 \\
\hline $\mathrm{C}$ & 8.307598 & 5.632204 & 15.435252 \\
\hline C & 6.710714 & 6.796778 & 9.284056 \\
\hline C & 3.851054 & 9.648609 & 18.285262 \\
\hline C & 2.594991 & 9.913584 & 12.055267 \\
\hline C & 13.517983 & 6.403842 & 16.007880 \\
\hline C & 14.785986 & 7.956546 & 9.885297 \\
\hline C & 15.474202 & 16.140142 & 16.156761 \\
\hline C & 14.471889 & 17.792430 & 10.150300 \\
\hline C & 6.347426 & 15.831016 & 6.796194 \\
\hline $\mathrm{C}$ & 10.195582 & 11.139499 & 5.961006 \\
\hline $\mathrm{H}$ & 7.679420 & 6.478547 & 15.731957 \\
\hline $\mathrm{H}$ & 8.998373 & 5.378943 & 16.248545 \\
\hline $\mathrm{H}$ & 7.681271 & 4.764225 & 15.196333 \\
\hline $\mathrm{H}$ & 7.759561 & 6.971269 & 9.024479 \\
\hline $\mathrm{H}$ & 6.061936 & 7.162899 & 8.478895 \\
\hline $\mathrm{H}$ & 6.536924 & 5.725465 & 9.443028 \\
\hline $\mathrm{H}$ & 3.849430 & 10.740611 & 18.349903 \\
\hline $\mathrm{H}$ & 4.280273 & 9.220339 & 19.199387 \\
\hline $\mathrm{H}$ & 2.826471 & 9.279322 & 18.151559 \\
\hline $\mathrm{H}$ & 3.325337 & 9.109321 & 11.919329 \\
\hline $\mathrm{H}$ & 2.326222 & 10.338812 & 11.080358 \\
\hline $\mathrm{H}$ & 1.696667 & 9.526206 & 12.551387 \\
\hline $\mathrm{H}$ & 12.495090 & 6.271028 & 15.638949 \\
\hline $\mathrm{H}$ & 13.519129 & 6.402623 & 17.105300 \\
\hline $\mathrm{H}$ & 14.158721 & 5.593745 & 15.638808 \\
\hline $\mathrm{H}$ & 15.283584 & 8.894030 & 10.152843 \\
\hline $\mathrm{H}$ & 14.465327 & 7.995431 & 8.836652 \\
\hline $\mathrm{H}$ & 15.466623 & 7.108758 & 10.030215 \\
\hline $\mathrm{H}$ & 15.852123 & 15.150463 & 15.879307 \\
\hline $\mathrm{H}$ & 15.281411 & 16.171531 & 17.236241 \\
\hline $\mathrm{H}$ & 16.204891 & 16.913120 & 15.888573 \\
\hline $\mathrm{H}$ & 13.470635 & 18.117965 & 10.450971 \\
\hline $\mathrm{H}$ & 14.542600 & 17.784673 & 9.056009 \\
\hline $\mathrm{H}$ & 15.227361 & 18.469214 & 10.567409 \\
\hline $\mathrm{H}$ & 6.639116 & 15.609740 & 5.762015 \\
\hline $\mathrm{H}$ & 5.992344 & 16.867643 & 6.858821 \\
\hline $\mathrm{H}$ & 5.554845 & 15.146699 & 7.114608 \\
\hline $\mathrm{H}$ & 9.942078 & 11.755293 & 5.090317 \\
\hline $\mathrm{H}$ & 10.322227 & 10.094396 & 5.649701 \\
\hline $\mathrm{H}$ & 11.123162 & 11.500597 & 6.419230 \\
\hline $\mathrm{Au}$ & 10.859672 & 12.079887 & 11.448220 \\
\hline $\mathrm{Au}$ & 5.462330 & 11.691094 & 12.257087 \\
\hline $\mathrm{Au}$ & 10.134762 & 13.725656 & 13.602226 \\
\hline $\mathrm{Au}$ & 7.621865 & 9.639028 & 14.110004 \\
\hline $\mathrm{Au}$ & 10.624055 & 10.975497 & 13.980761 \\
\hline $\mathrm{Au}$ & 8.302405 & 11.848576 & 12.531471 \\
\hline $\mathrm{Au}$ & 12.484934 & 14.626031 & 15.801756 \\
\hline $\mathrm{Au}$ & 7.871631 & 14.651087 & 15.250757 \\
\hline $\mathrm{Au}$ & 12.346486 & 9.563103 & 15.595194 \\
\hline $\mathrm{Au}$ & 12.795737 & 14.937299 & 10.241199 \\
\hline $\mathrm{Au}$ & 11.932663 & 9.620936 & 10.660721 \\
\hline $\mathrm{Au}$ & 7.331697 & 15.435747 & 10.234870 \\
\hline $\mathrm{Au}$ & 6.902085 & 10.244268 & 16.873518 \\
\hline $\mathrm{Au}$ & 6.934744 & 9.958107 & 10.561529 \\
\hline $\mathrm{Au}$ & 14.304474 & 16.177344 & 13.031053 \\
\hline
\end{tabular}




\begin{tabular}{|c|c|c|c|}
\hline $\mathrm{Au}$ & 13.818395 & 7.975336 & 13.143260 \\
\hline $\mathrm{Au}$ & 11.230338 & 12.324359 & 16.523910 \\
\hline $\mathrm{Au}$ & 11.247989 & 13.067839 & 8.868230 \\
\hline $\mathrm{Au}$ & 8.712070 & 16.107925 & 12.557768 \\
\hline $\mathrm{Au}$ & 7.814792 & 6.991481 & 12.413934 \\
\hline $\mathrm{Au}$ & 8.394039 & 12.526742 & 16.985496 \\
\hline $\mathrm{Au}$ & 12.950797 & 13.537015 & 12.946815 \\
\hline $\mathrm{Au}$ & 6.786302 & 14.216664 & 12.679179 \\
\hline $\mathrm{Au}$ & 9.964732 & 14.752674 & 17.172919 \\
\hline $\mathrm{Au}$ & 10.061776 & 15.008691 & 10.349662 \\
\hline $\mathrm{Au}$ & 13.451257 & 10.790377 & 12.857033 \\
\hline $\mathrm{Au}$ & 4.149693 & 10.238823 & 14.981933 \\
\hline $\mathrm{Au}$ & 9.604460 & 10.092583 & 16.406573 \\
\hline $\mathrm{Au}$ & 9.699060 & 10.615359 & 9.295871 \\
\hline $\mathrm{Au}$ & 13.647908 & 12.114104 & 15.258815 \\
\hline $\mathrm{Au}$ & 6.594839 & 12.236888 & 14.820697 \\
\hline $\mathrm{Au}$ & 10.175204 & 16.257809 & 14.904142 \\
\hline $\mathrm{Au}$ & 10.160501 & 8.224587 & 14.373400 \\
\hline $\mathrm{Au}$ & 13.422066 & 12.118199 & 10.392533 \\
\hline $\mathrm{Au}$ & 7.121435 & 12.634334 & 10.139056 \\
\hline $\mathrm{Au}$ & 11.501293 & 16.061491 & 12.531554 \\
\hline $\mathrm{Au}$ & 9.419839 & 9.353058 & 11.827347 \\
\hline $\mathrm{Au}$ & 8.457405 & 13.433563 & 7.694391 \\
\hline \multicolumn{4}{|c|}{ \#figure 3c } \\
\hline $\mathrm{S}$ & 15.377809 & 13.575110 & 16.045981 \\
\hline $\mathrm{s}$ & 17.136932 & 11.059946 & 13.210886 \\
\hline $\mathrm{S}$ & 5.108548 & 11.128162 & 11.144998 \\
\hline $\mathrm{S}$ & 5.256349 & 17.195271 & 14.255142 \\
\hline $\mathrm{S}$ & 15.178976 & 10.891521 & 8.739050 \\
\hline $\mathrm{S}$ & 11.226490 & 4.962123 & 9.208303 \\
\hline $\mathrm{S}$ & 6.718729 & 8.854196 & 7.609774 \\
\hline $\mathrm{S}$ & 14.560460 & 5.236804 & 12.464682 \\
\hline $\mathrm{S}$ & 8.994723 & 16.529486 & 9.595551 \\
\hline $\mathrm{S}$ & 8.268816 & 17.819586 & 17.750291 \\
\hline $\mathrm{S}$ & 11.963592 & 7.322581 & 15.703379 \\
\hline $\mathrm{s}$ & 9.301672 & 12.307254 & 5.902335 \\
\hline $\mathrm{S}$ & 7.279197 & 8.437063 & 16.310621 \\
\hline $\mathrm{S}$ & 13.035411 & 13.711651 & 20.046249 \\
\hline C & 15.829127 & 15.250620 & 15.468440 \\
\hline C & 16.817606 & 10.215500 & 14.788914 \\
\hline C & 3.751129 & 10.125160 & 11.852814 \\
\hline C & 3.655957 & 16.879053 & 15.087935 \\
\hline C & 16.887310 & 10.441823 & 9.194874 \\
\hline C & 9.896777 & 3.943239 & 9.945667 \\
\hline C & 7.052591 & 7.412419 & 6.523361 \\
\hline C & 15.831753 & 6.412597 & 11.868779 \\
\hline C & 10.452716 & 17.576347 & 9.229893 \\
\hline C & 9.368890 & 19.194028 & 17.232147 \\
\hline C & 10.799693 & 5.984882 & 16.157420 \\
\hline $\mathrm{C}$ & 8.307214 & 13.841677 & 5.810685 \\
\hline C & 5.490693 & 8.838156 & 16.354736 \\
\hline C & 13.308633 & 15.267605 & 20.976926 \\
\hline $\mathrm{H}$ & 16.201296 & 15.143612 & 14.441596 \\
\hline $\mathrm{H}$ & 16.631481 & 15.631345 & 16.112249 \\
\hline $\mathrm{H}$ & 14.971347 & 15.930082 & 15.487103 \\
\hline $\mathrm{H}$ & 16.474156 & 10.922058 & 15.554555 \\
\hline $\mathrm{H}$ & 17.780113 & 9.787317 & 15.105553 \\
\hline $\mathrm{H}$ & 16.088413 & 9.404245 & 14.671621 \\
\hline $\mathrm{H}$ & 3.990525 & 9.773720 & 12.860855 \\
\hline $\mathrm{H}$ & 3.579315 & 9.269936 & 11.187723 \\
\hline $\mathrm{H}$ & 2.853503 & 10.755762 & 11.882265 \\
\hline $\mathrm{H}$ & 2.951880 & 16.524625 & 14.324989 \\
\hline $\mathrm{H}$ & 3.293867 & 17.823380 & 15.512490 \\
\hline $\mathrm{H}$ & 3.753803 & 16.127760 & 15.877145 \\
\hline $\mathrm{H}$ & 17.575110 & 11.057186 & 8.600864 \\
\hline $\mathrm{H}$ & 17.038750 & 9.383801 & 8.944119 \\
\hline $\mathrm{H}$ & 17.080064 & 10.594338 & 10.263843 \\
\hline $\mathrm{H}$ & 9.033359 & 3.985667 & 9.270711 \\
\hline $\mathrm{H}$ & 9.609157 & 4.305640 & 10.937023 \\
\hline $\mathrm{H}$ & 10.259860 & 2.910636 & 10.015199 \\
\hline
\end{tabular}




\begin{tabular}{|c|c|c|c|}
\hline $\mathrm{H}$ & 8.106933 & 7.358236 & 6.236628 \\
\hline $\mathrm{H}$ & 6.426938 & 7.509438 & 5.627757 \\
\hline $\mathrm{H}$ & 6.770427 & 6.505993 & 7.073107 \\
\hline $\mathrm{H}$ & 16.550019 & 6.567386 & 12.682651 \\
\hline $\mathrm{H}$ & 15.389127 & 7.370498 & 11.574050 \\
\hline $\mathrm{H}$ & 16.336015 & 5.948958 & 11.012698 \\
\hline $\mathrm{H}$ & 11.277196 & 17.365309 & 9.918525 \\
\hline $\mathrm{H}$ & 10.144897 & 18.624521 & 9.334136 \\
\hline $\mathrm{H}$ & 10.769338 & 17.379930 & 8.199017 \\
\hline $\mathrm{H}$ & 10.185360 & 19.266549 & 17.960346 \\
\hline $\mathrm{H}$ & 8.777067 & 20.117225 & 17.244935 \\
\hline $\mathrm{H}$ & 9.771465 & 19.021002 & 16.229417 \\
\hline $\mathrm{H}$ & 11.374602 & 5.204347 & 16.671489 \\
\hline $\mathrm{H}$ & 10.300777 & 5.564647 & 15.278896 \\
\hline $\mathrm{H}$ & 10.053289 & 6.408227 & 16.840442 \\
\hline $\mathrm{H}$ & 7.674699 & 13.784335 & 4.916958 \\
\hline $\mathrm{H}$ & 9.005744 & 14.682449 & 5.717612 \\
\hline $\mathrm{H}$ & 7.687364 & 13.977231 & 6.701835 \\
\hline $\mathrm{H}$ & 5.225648 & 9.066579 & 17.394644 \\
\hline $\mathrm{H}$ & 4.929462 & 7.958174 & 16.018068 \\
\hline $\mathrm{H}$ & 5.262066 & 9.701845 & 15.721146 \\
\hline $\mathrm{H}$ & 14.251342 & 15.170291 & 21.529434 \\
\hline $\mathrm{H}$ & 12.477786 & 15.380768 & 21.683375 \\
\hline $\mathrm{H}$ & 13.355464 & 16.138057 & 20.316000 \\
\hline $\mathrm{Au}$ & 11.287683 & 14.496493 & 15.596198 \\
\hline $\mathrm{Au}$ & 11.168187 & 12.877072 & 13.033731 \\
\hline $\mathrm{Au}$ & 8.521984 & 13.750026 & 12.749769 \\
\hline $\mathrm{Au}$ & 12.354813 & 8.167027 & 11.853753 \\
\hline $\mathrm{Au}$ & 9.355299 & 13.026981 & 16.982399 \\
\hline $\mathrm{Au}$ & 9.378184 & 11.806465 & 10.913725 \\
\hline $\mathrm{Au}$ & 11.346153 & 15.612856 & 13.110648 \\
\hline $\mathrm{Au}$ & 7.323509 & 12.846676 & 15.161762 \\
\hline $\mathrm{Au}$ & 11.165138 & 11.462245 & 15.485243 \\
\hline $\mathrm{Au}$ & 13.426185 & 14.231039 & 11.925855 \\
\hline $\mathrm{Au}$ & 12.781326 & 10.557998 & 13.211767 \\
\hline $\mathrm{Au}$ & 9.096170 & 11.056112 & 13.565686 \\
\hline $\mathrm{Au}$ & 9.466522 & 8.664873 & 11.761030 \\
\hline $\mathrm{Au}$ & 11.131998 & 9.910622 & 9.815753 \\
\hline $\mathrm{Au}$ & 9.840829 & 14.355336 & 9.936230 \\
\hline $\mathrm{Au}$ & 13.229868 & 6.406451 & 13.983261 \\
\hline $\mathrm{Au}$ & 9.542044 & 15.859667 & 17.332933 \\
\hline $\mathrm{Au}$ & 14.710044 & 12.926717 & 9.955766 \\
\hline Au & 6.230156 & 15.040205 & 13.956957 \\
\hline $\mathrm{Au}$ & 10.698958 & 7.222878 & 9.662345 \\
\hline $\mathrm{Au}$ & 8.328294 & 10.614357 & 16.421890 \\
\hline $\mathrm{Au}$ & 10.100463 & 12.100224 & 8.168840 \\
\hline $\mathrm{Au}$ & 13.482880 & 12.942445 & 14.680178 \\
\hline $\mathrm{Au}$ & 5.898587 & 12.577059 & 12.833860 \\
\hline $\mathrm{Au}$ & 8.932040 & 16.045528 & 14.587673 \\
\hline $\mathrm{Au}$ & 11.995045 & 12.585722 & 10.116431 \\
\hline $\mathrm{Au}$ & 11.473150 & 14.201427 & 18.315368 \\
\hline $\mathrm{Au}$ & 8.623463 & 9.021878 & 9.063692 \\
\hline $\mathrm{Au}$ & 7.913726 & 8.530610 & 14.009720 \\
\hline $\mathrm{Au}$ & 13.838936 & 10.161027 & 10.652466 \\
\hline $\mathrm{Au}$ & 14.326916 & 13.778393 & 18.088309 \\
\hline $\mathrm{Au}$ & 7.095175 & 9.963774 & 11.691356 \\
\hline $\mathrm{Au}$ & 6.804371 & 17.502865 & 15.974061 \\
\hline Au & 10.742776 & 8.884441 & 14.359588 \\
\hline $\mathrm{Au}$ & 15.228493 & 12.144457 & 12.541259 \\
\hline $\mathrm{Au}$ & 7.900783 & 10.615667 & 6.647065 \\
\hline $\mathrm{Au}$ & 8.903880 & 16.449420 & 11.970090 \\
\hline $\mathrm{Au}$ & 12.853478 & 5.244196 & 10.874141 \\
\hline \multicolumn{4}{|c|}{ \#figure 3d } \\
\hline S & 8.312283 & 5.501914 & 13.344757 \\
\hline S & 5.625472 & 7.639780 & 10.187319 \\
\hline S & 5.930934 & 8.787234 & 17.425436 \\
\hline S & 4.164130 & 10.878443 & 13.659053 \\
\hline S & 13.248362 & 6.925901 & 14.625724 \\
\hline S & 11.046353 & 7.494696 & 10.541190 \\
\hline S & 11.516271 & 12.649683 & 6.839396 \\
\hline
\end{tabular}




\begin{tabular}{|c|c|c|}
\hline 7.908837 & 15.592867 & 6.828454 \\
\hline 15.996947 & 12.997930 & 14.243479 \\
\hline 14.467186 & 12.169769 & 18.564171 \\
\hline 14.534076 & 16.733922 & 11.391712 \\
\hline 13.387281 & 16.832274 & 15.886817 \\
\hline 10.040484 & 18.346326 & 13.207001 \\
\hline 9.069052 & 15.902333 & 17.055142 \\
\hline 5.420088 & 15.934278 & 14.422908 \\
\hline 3.954781 & 14.116967 & 10.363897 \\
\hline 15.032921 & 9.554376 & 11.482546 \\
\hline 14.127662 & 13.558570 & 9.293765 \\
\hline 8.032057 & 12.856063 & 18.224751 \\
\hline 10.035894 & 8.554221 & 17.549637 \\
\hline 8.909289 & 8.470366 & 7.368648 \\
\hline 6.336711 & 12.316489 & 7.271446 \\
\hline 7.188277 & 5.099119 & 14.731810 \\
\hline 5.983949 & 6.701266 & 8.662421 \\
\hline 4.498433 & 8.989534 & 18.560240 \\
\hline 3.389321 & 9.499752 & 12.734811 \\
\hline 13.225912 & 5.103581 & 14.811540 \\
\hline 12.414099 & 7.414880 & 9.334757 \\
\hline 11.157403 & 11.250941 & 5.718740 \\
\hline 8.662065 & 17.255411 & 6.704449 \\
\hline 16.835028 & 14.612631 & 14.120381 \\
\hline 14.708596 & 10.358640 & 18.715243 \\
\hline 14.013889 & 18.308803 & 10.622851 \\
\hline 14.903125 & 16.515488 & 16.868217 \\
\hline 8.485935 & 18.475986 & 12.239936 \\
\hline 10.257296 & 16.524208 & 18.302537 \\
\hline 4.363557 & 15.173181 & 15.711067 \\
\hline 2.570036 & 13.030265 & 10.848878 \\
\hline 16.495980 & 9.706450 & 12.568409 \\
\hline 15.703527 & 14.440443 & 9.024724 \\
\hline 9.020160 & 12.590670 & 19.737594 \\
\hline 10.838945 & 8.488987 & 19.194016 \\
\hline 8.484847 & 7.729347 & 5.752788 \\
\hline 4.726805 & 11.876489 & 6.524501 \\
\hline 6.653558 & 5.985073 & 15.090576 \\
\hline 7.797843 & 4.684606 & 15.543848 \\
\hline 6.471304 & 4.344418 & 14.385749 \\
\hline 7.018295 & 6.884925 & 8.340290 \\
\hline 5.289101 & 7.061377 & 7.892428 \\
\hline 5.808302 & 5.636442 & 8.855544 \\
\hline 4.080017 & 9.999024 & 18.505546 \\
\hline 4.850381 & 8.786671 & 19.579066 \\
\hline 3.736372 & 8.252501 & 18.279241 \\
\hline 4.119417 & 8.723575 & 12.483369 \\
\hline 2.966356 & 9.908377 & 11.808756 \\
\hline 2.587139 & 9.075856 & 13.351044 \\
\hline 12.217620 & 4.698096 & 14.682547 \\
\hline 13.604265 & 4.860543 & 15.812086 \\
\hline 13.897013 & 4.683706 & 14.052475 \\
\hline 13.237837 & 8.083599 & 9.606108 \\
\hline 11.992384 & 7.707147 & 8.364114 \\
\hline 12.772057 & 6.378751 & 9.291128 \\
\hline 10.229939 & 10.735824 & 5.996143 \\
\hline 11.998098 & 10.549829 & 5.797383 \\
\hline 11.093500 & 11.640494 & 4.695411 \\
\hline 9.540174 & 17.352363 & 7.350153 \\
\hline 7.904340 & 17.993095 & 6.997642 \\
\hline 8.950915 & 17.423883 & 5.659438 \\
\hline 16.139011 & 15.444731 & 14.296445 \\
\hline 17.240024 & 14.697057 & 13.104254 \\
\hline 17.654685 & 14.636489 & 14.848912 \\
\hline 14.319414 & 9.824162 & 17.842735 \\
\hline 14.183852 & 10.027904 & 19.620726 \\
\hline 15.781943 & 10.162229 & 18.824605 \\
\hline 12.977915 & 18.558539 & 10.874259 \\
\hline 14.110057 & 18.195233 & 9.535568 \\
\hline 14.682911 & 19.107405 & 10.967175 \\
\hline 15.373017 & 15.562538 & 16.594998 \\
\hline
\end{tabular}

S15 


\begin{tabular}{|c|c|c|c|}
\hline $\mathrm{H}$ & 14.607121 & 16.483371 & 17.924333 \\
\hline $\mathrm{H}$ & 15.597609 & 17.348099 & 16.703336 \\
\hline $\mathrm{H}$ & 8.069756 & 17.487429 & 12.018560 \\
\hline $\mathrm{H}$ & 8.723100 & 18.996699 & 11.304326 \\
\hline $\mathrm{H}$ & 7.769225 & 19.067462 & 12.822456 \\
\hline $\mathrm{H}$ & 11.288145 & 16.465117 & 17.936514 \\
\hline $\mathrm{H}$ & 10.144237 & 15.909937 & 19.204983 \\
\hline $\mathrm{H}$ & 10.002290 & 17.565796 & 18.535751 \\
\hline $\mathrm{H}$ & 3.362689 & 15.615993 & 15.632774 \\
\hline $\mathrm{H}$ & 4.800826 & 15.417259 & 16.686758 \\
\hline $\mathrm{H}$ & 4.299337 & 14.086732 & 15.592780 \\
\hline $\mathrm{H}$ & 2.854664 & 12.355366 & 11.664103 \\
\hline $\mathrm{H}$ & 2.273422 & 12.447608 & 9.967217 \\
\hline $\mathrm{H}$ & 1.729098 & 13.656913 & 11.171359 \\
\hline $\mathrm{H}$ & 17.391094 & 9.640716 & 11.937415 \\
\hline $\mathrm{H}$ & 16.477104 & 8.862488 & 13.269277 \\
\hline $\mathrm{H}$ & 16.499143 & 10.652362 & 13.120164 \\
\hline $\mathrm{H}$ & 16.229476 & 14.638327 & 9.964124 \\
\hline $\mathrm{H}$ & 15.472092 & 15.395116 & 8.535127 \\
\hline $\mathrm{H}$ & 16.330125 & 13.831639 & 8.361861 \\
\hline $\mathrm{H}$ & 8.352803 & 12.287278 & 20.553663 \\
\hline $\mathrm{H}$ & 9.498541 & 13.544729 & 19.995288 \\
\hline $\mathrm{H}$ & 9.796496 & 11.834470 & 19.575608 \\
\hline $\mathrm{H}$ & 10.169944 & 8.987533 & 19.907529 \\
\hline $\mathrm{H}$ & 11.812932 & 8.987751 & 19.188267 \\
\hline $\mathrm{H}$ & 10.956894 & 7.436096 & 19.478152 \\
\hline $\mathrm{H}$ & 7.412271 & 7.507652 & 5.679501 \\
\hline $\mathrm{H}$ & 9.041575 & 6.784704 & 5.677435 \\
\hline $\mathrm{H}$ & 8.775081 & 8.377577 & 4.915980 \\
\hline $\mathrm{H}$ & 4.904206 & 11.500945 & 5.509580 \\
\hline $\mathrm{H}$ & 4.122576 & 12.791810 & 6.479826 \\
\hline $\mathrm{H}$ & 4.204164 & 11.116090 & 7.114049 \\
\hline $\mathrm{Au}$ & 11.517297 & 12.304920 & 11.799859 \\
\hline $\mathrm{Au}$ & 6.054690 & 11.524373 & 12.266568 \\
\hline $\mathrm{Au}$ & 10.068908 & 14.409545 & 13.674153 \\
\hline $\mathrm{Au}$ & 8.064059 & 9.808187 & 15.153814 \\
\hline $\mathrm{Au}$ & 10.533544 & 9.999871 & 13.715443 \\
\hline $\mathrm{Au}$ & 8.568773 & 11.791762 & 10.704313 \\
\hline $\mathrm{Au}$ & 11.735958 & 12.253608 & 14.657136 \\
\hline $\mathrm{Au}$ & 7.415443 & 14.652114 & 14.406307 \\
\hline $\mathrm{Au}$ & 11.577934 & 7.730926 & 16.021006 \\
\hline $\mathrm{Au}$ & 12.852304 & 14.807006 & 10.999676 \\
\hline $\mathrm{Au}$ & 10.545958 & 9.824873 & 10.715520 \\
\hline $\mathrm{Au}$ & 7.934554 & 14.945957 & 9.125670 \\
\hline $\mathrm{Au}$ & 7.147536 & 10.779283 & 17.726475 \\
\hline $\mathrm{Au}$ & 6.713989 & 9.785487 & 10.020150 \\
\hline $\mathrm{Au}$ & 13.946380 & 16.843613 & 13.629594 \\
\hline $\mathrm{Au}$ & 12.080557 & 7.339220 & 12.630144 \\
\hline $\mathrm{Au}$ & 12.436679 & 12.479312 & 17.349126 \\
\hline $\mathrm{Au}$ & 10.738325 & 12.029283 & 9.018073 \\
\hline $\mathrm{Au}$ & 7.384925 & 14.158338 & 11.673899 \\
\hline $\mathrm{Au}$ & 7.029668 & 6.715236 & 11.797097 \\
\hline $\mathrm{Au}$ & 9.837559 & 13.510128 & 16.557675 \\
\hline $\mathrm{Au}$ & 13.911924 & 12.973476 & 13.065641 \\
\hline $\mathrm{Au}$ & 4.720112 & 14.965709 & 12.402023 \\
\hline $\mathrm{Au}$ & 12.174186 & 14.810033 & 15.700384 \\
\hline $\mathrm{Au}$ & 10.030434 & 14.376591 & 10.802535 \\
\hline $\mathrm{Au}$ & 13.060384 & 10.165096 & 12.757069 \\
\hline $\mathrm{Au}$ & 5.191150 & 9.815590 & 15.447553 \\
\hline $\mathrm{Au}$ & 10.303852 & 10.799142 & 16.702536 \\
\hline $\mathrm{Au}$ & 7.574377 & 10.354348 & 7.401782 \\
\hline $\mathrm{Au}$ & 15.268338 & 12.681280 & 16.415233 \\
\hline $\mathrm{Au}$ & 8.475599 & 12.173594 & 13.738081 \\
\hline $\mathrm{Au}$ & 9.556646 & 17.018593 & 15.071057 \\
\hline $\mathrm{Au}$ & 9.360555 & 7.553998 & 13.948323 \\
\hline $\mathrm{Au}$ & 14.666576 & 11.637711 & 10.471738 \\
\hline $\mathrm{Au}$ & 5.951472 & 12.681008 & 9.652198 \\
\hline $\mathrm{Au}$ & 11.244799 & 16.488415 & 12.296284 \\
\hline $\mathrm{Au}$ & 8.026088 & 9.482116 & 12.414675 \\
\hline $\mathrm{Au}$ & 9.703763 & 14.095206 & 6.749249 \\
\hline
\end{tabular}




\begin{tabular}{|c|c|c|c|}
\hline \multicolumn{4}{|c|}{ \#figure $4 a$} \\
\hline $\mathrm{S}$ & 15.847544 & 14.079491 & 14.375349 \\
\hline $\mathrm{S}$ & 15.701532 & 10.009798 & 10.575069 \\
\hline $\mathrm{s}$ & 4.302088 & 13.588784 & 14.102035 \\
\hline $\mathrm{S}$ & 8.908910 & 17.835844 & 14.280469 \\
\hline $\mathrm{S}$ & 11.806595 & 10.738515 & 6.788114 \\
\hline $\mathrm{S}$ & 7.619437 & 6.724087 & 10.987399 \\
\hline $\mathrm{S}$ & 4.119894 & 10.560287 & 11.544289 \\
\hline $\mathrm{S}$ & 11.604439 & 6.432781 & 11.080623 \\
\hline $\mathrm{S}$ & 12.189445 & 17.473334 & 11.155226 \\
\hline $\mathrm{S}$ & 11.606926 & 13.394043 & 18.740251 \\
\hline $\mathrm{S}$ & 11.682436 & 6.473285 & 15.149464 \\
\hline $\mathrm{S}$ & 8.913741 & 13.408072 & 6.779643 \\
\hline $\mathrm{S}$ & 8.889373 & 10.324870 & 18.653749 \\
\hline S & 15.892181 & 10.153428 & 14.625970 \\
\hline $\mathrm{C}$ & 15.451946 & 15.624120 & 15.265499 \\
\hline $\mathrm{C}$ & 17.381118 & 10.479600 & 11.124228 \\
\hline $\mathrm{C}$ & 4.026842 & 14.952656 & 15.286587 \\
\hline $\mathrm{C}$ & 7.557811 & 18.083184 & 15.486275 \\
\hline $\mathrm{C}$ & 13.170682 & 9.550114 & 6.524604 \\
\hline $\mathrm{C}$ & 8.134553 & 5.018044 & 11.406993 \\
\hline $\mathrm{C}$ & 3.990039 & 9.161724 & 10.369332 \\
\hline $\mathrm{C}$ & 12.908828 & 6.520709 & 9.795112 \\
\hline $\mathrm{C}$ & 11.517469 & 19.013415 & 11.873612 \\
\hline $\mathrm{C}$ & 12.766164 & 14.790723 & 18.948104 \\
\hline $\mathrm{C}$ & 11.248845 & 4.852322 & 14.431814 \\
\hline $\mathrm{C}$ & 8.119956 & 14.928414 & 6.151715 \\
\hline $\mathrm{C}$ & 7.713944 & 8.931237 & 18.830273 \\
\hline $\mathrm{C}$ & 15.617614 & 8.874315 & 15.899242 \\
\hline $\mathrm{H}$ & 14.375184 & 15.840529 & 15.238953 \\
\hline $\mathrm{H}$ & 16.004626 & 16.445706 & 14.791584 \\
\hline $\mathrm{H}$ & 15.784554 & 15.508743 & 16.304771 \\
\hline $\mathrm{H}$ & 17.895907 & 11.003330 & 10.309235 \\
\hline $\mathrm{H}$ & 17.915781 & 9.550889 & 11.360039 \\
\hline $\mathrm{H}$ & 17.349992 & 11.113990 & 12.016885 \\
\hline $\mathrm{H}$ & 3.562453 & 15.794211 & 14.757217 \\
\hline $\mathrm{H}$ & 4.964429 & 15.274862 & 15.753072 \\
\hline $\mathrm{H}$ & 3.341778 & 14.585397 & 16.062220 \\
\hline $\mathrm{H}$ & 7.171361 & 17.124601 & 15.849554 \\
\hline $\mathrm{H}$ & 6.755571 & 18.649013 & 14.996637 \\
\hline $\mathrm{H}$ & 7.956442 & 18.662649 & 16.328014 \\
\hline $\mathrm{H}$ & 12.749996 & 8.664519 & 6.032845 \\
\hline $\mathrm{H}$ & 13.642590 & 9.260115 & 7.468680 \\
\hline $\mathrm{H}$ & 13.913380 & 10.020696 & 5.869241 \\
\hline $\mathrm{H}$ & 7.393564 & 4.337525 & 10.967655 \\
\hline $\mathrm{H}$ & 8.159905 & 4.873283 & 12.492234 \\
\hline $\mathrm{H}$ & 9.126983 & 4.815017 & 10.984553 \\
\hline $\mathrm{H}$ & 4.977576 & 8.794613 & 10.067986 \\
\hline $\mathrm{H}$ & 3.446860 & 9.524479 & 9.486715 \\
\hline $\mathrm{H}$ & 3.422312 & 8.355397 & 10.847459 \\
\hline $\mathrm{H}$ & 13.691351 & 7.236466 & 10.072030 \\
\hline $\mathrm{H}$ & 12.464612 & 6.814225 & 8.838191 \\
\hline $\mathrm{H}$ & 13.347447 & 5.518357 & 9.699859 \\
\hline $\mathrm{H}$ & 11.133123 & 19.621143 & 11.043694 \\
\hline $\mathrm{H}$ & 12.339946 & 19.544800 & 12.367544 \\
\hline $\mathrm{H}$ & 10.712110 & 18.826475 & 12.592570 \\
\hline $\mathrm{H}$ & 13.097256 & 15.188043 & 17.984802 \\
\hline $\mathrm{H}$ & 13.630123 & 14.423528 & 19.515889 \\
\hline $\mathrm{H}$ & 12.259651 & 15.577026 & 19.520665 \\
\hline $\mathrm{H}$ & 10.570843 & 4.949331 & 13.576990 \\
\hline $\mathrm{H}$ & 10.776690 & 4.247055 & 15.216445 \\
\hline $\mathrm{H}$ & 12.175269 & 4.364865 & 14.099965 \\
\hline $\mathrm{H}$ & 8.862574 & 15.484181 & 5.565863 \\
\hline $\mathrm{H}$ & 7.749998 & 15.556342 & 6.968310 \\
\hline $\mathrm{H}$ & 7.284676 & 14.640245 & 5.500547 \\
\hline $\mathrm{H}$ & 6.690295 & 9.288148 & 18.675062 \\
\hline $\mathrm{H}$ & 7.823506 & 8.548823 & 19.854063 \\
\hline $\mathrm{H}$ & 7.941260 & 8.137503 & 18.111815 \\
\hline $\mathrm{H}$ & 16.215179 & 9.145820 & 16.778759 \\
\hline $\mathrm{H}$ & 15.956207 & 7.907800 & 15.507019 \\
\hline $\mathrm{H}$ & 14.560341 & 8.803972 & 16.185877 \\
\hline
\end{tabular}




\begin{tabular}{|c|c|c|c|}
\hline $\mathrm{Au}$ & 12.414018 & 11.953580 & 12.817974 \\
\hline $\mathrm{Au}$ & 7.950160 & 11.947159 & 12.694323 \\
\hline $\mathrm{Au}$ & 10.172355 & 13.892318 & 12.843980 \\
\hline $\mathrm{Au}$ & 9.954095 & 9.953927 & 12.812745 \\
\hline $\mathrm{Au}$ & 10.216147 & 11.892923 & 14.829971 \\
\hline $\mathrm{Au}$ & 10.338827 & 11.961196 & 10.795064 \\
\hline $\mathrm{Au}$ & 12.268225 & 13.991531 & 14.904154 \\
\hline $\mathrm{Au}$ & 8.167784 & 13.900390 & 15.015538 \\
\hline $\mathrm{Au}$ & 12.430047 & 9.998468 & 14.911390 \\
\hline $\mathrm{Au}$ & 12.436402 & 13.950578 & 10.591472 \\
\hline $\mathrm{Au}$ & 12.143997 & 9.673216 & 11.075977 \\
\hline $\mathrm{Au}$ & 8.112769 & 14.155586 & 10.811171 \\
\hline $\mathrm{Au}$ & 7.846955 & 9.945379 & 14.918299 \\
\hline $\mathrm{Au}$ & 8.228715 & 10.256420 & 10.155810 \\
\hline $\mathrm{Au}$ & 12.357005 & 15.898107 & 12.958458 \\
\hline $\mathrm{Au}$ & 12.218165 & 7.826221 & 13.134587 \\
\hline $\mathrm{Au}$ & 12.373083 & 11.939461 & 16.946852 \\
\hline $\mathrm{Au}$ & 12.515143 & 12.224780 & 8.476239 \\
\hline $\mathrm{Au}$ & 8.182925 & 16.118784 & 12.739512 \\
\hline $\mathrm{Au}$ & 7.805069 & 8.042836 & 12.934013 \\
\hline $\mathrm{Au}$ & 7.970802 & 11.791532 & 16.968160 \\
\hline $\mathrm{Au}$ & 7.583110 & 12.574534 & 8.589657 \\
\hline $\mathrm{Au}$ & 14.478519 & 13.991260 & 12.426564 \\
\hline $\mathrm{Au}$ & 6.136431 & 14.214773 & 12.707551 \\
\hline $\mathrm{Au}$ & 10.059010 & 13.950589 & 16.987954 \\
\hline $\mathrm{Au}$ & 10.221177 & 13.963659 & 8.767573 \\
\hline $\mathrm{Au}$ & 14.323220 & 9.777320 & 12.777023 \\
\hline $\mathrm{Au}$ & 5.761893 & 10.023800 & 13.142735 \\
\hline $\mathrm{Au}$ & 10.412161 & 9.909416 & 16.845337 \\
\hline $\mathrm{Au}$ & 10.643471 & 9.880386 & 8.703207 \\
\hline $\mathrm{Au}$ & 14.437671 & 12.149105 & 15.034414 \\
\hline $\mathrm{Au}$ & 6.002726 & 12.094979 & 15.051065 \\
\hline $\mathrm{Au}$ & 10.281377 & 15.933156 & 14.930700 \\
\hline $\mathrm{Au}$ & 9.812607 & 7.969958 & 14.872700 \\
\hline $\mathrm{Au}$ & 14.391191 & 11.976423 & 10.533036 \\
\hline $\mathrm{Au}$ & 5.970557 & 11.793258 & 10.546995 \\
\hline $\mathrm{Au}$ & 10.361011 & 15.910223 & 10.730908 \\
\hline $\mathrm{Au}$ & 9.761456 & 8.030063 & 10.528045 \\
\hline \multicolumn{4}{|c|}{ \#figure 4b } \\
\hline $\mathrm{S}$ & 15.837750 & 14.939500 & 15.016650 \\
\hline $\mathrm{S}$ & 17.023100 & 11.347425 & 13.328600 \\
\hline $\mathrm{S}$ & 3.295300 & 10.722200 & 13.586050 \\
\hline $\mathrm{S}$ & 5.871650 & 17.802250 & 12.908550 \\
\hline $\mathrm{S}$ & 12.308375 & 9.311625 & 7.564550 \\
\hline $\mathrm{S}$ & 8.969100 & 4.603925 & 11.890975 \\
\hline $\mathrm{S}$ & 5.400975 & 9.435525 & 9.543250 \\
\hline $\mathrm{S}$ & 13.704450 & 4.569125 & 11.844025 \\
\hline $\mathrm{S}$ & 12.129000 & 18.055425 & 9.336050 \\
\hline $\mathrm{S}$ & 10.465250 & 16.392075 & 16.068425 \\
\hline $\mathrm{S}$ & 12.687325 & 5.846400 & 16.133525 \\
\hline $\mathrm{S}$ & 9.219325 & 14.110300 & 7.217700 \\
\hline $\mathrm{S}$ & 7.619650 & 10.521975 & 17.516025 \\
\hline $\mathrm{S}$ & 13.861000 & 11.325575 & 17.191450 \\
\hline C & 15.165750 & 16.324650 & 15.952000 \\
\hline C & 18.480200 & 11.820400 & 12.314150 \\
\hline C & 2.025350 & 11.484500 & 14.653400 \\
\hline C & 5.935900 & 17.862350 & 14.862600 \\
\hline C & 13.833700 & 8.273125 & 7.360575 \\
\hline $\mathrm{C}$ & 8.584350 & 4.836325 & 13.633000 \\
\hline C & 6.040575 & 8.201850 & 8.351000 \\
\hline $\mathrm{C}$ & 14.378350 & 5.680825 & 10.600575 \\
\hline C & 13.318175 & 19.215325 & 10.277925 \\
\hline $\mathrm{C}$ & 11.215850 & 18.064925 & 15.628550 \\
\hline $\mathrm{C}$ & 11.478425 & 4.457050 & 16.414275 \\
\hline C & 9.507300 & 15.952650 & 7.015975 \\
\hline C & 6.404575 & 9.074250 & 17.872575 \\
\hline C & 14.637450 & 11.678700 & 18.843850 \\
\hline $\mathrm{H}$ & 16.013500 & 16.870750 & 16.282575 \\
\hline $\mathrm{H}$ & 14.616775 & 15.788875 & 16.727025 \\
\hline $\mathrm{H}$ & 14.530025 & 17.017400 & 15.375400 \\
\hline
\end{tabular}




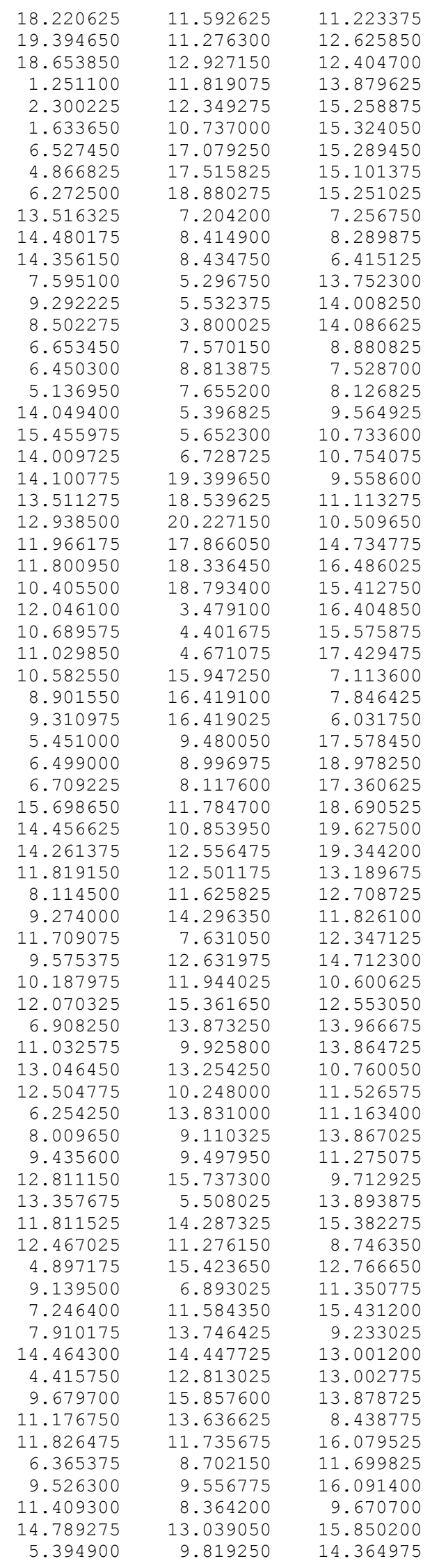




\begin{tabular}{|c|c|c|c|}
\hline $\mathrm{Au}$ & 7.853575 & 16.549550 & 12.152525 \\
\hline $\mathrm{Au}$ & 11.327825 & 7.607525 & 15.404100 \\
\hline $\mathrm{Au}$ & 14.804700 & 11.617425 & 12.443075 \\
\hline $\mathrm{Au}$ & 6.839250 & 11.384200 & 9.893050 \\
\hline $\mathrm{Au}$ & 10.380025 & 17.006325 & 10.965825 \\
\hline $\mathrm{Au}$ & 11.251050 & 4.578825 & 11.724800 \\
\hline \multicolumn{4}{|c|}{ \#figure 4c } \\
\hline S & 15.377809 & 13.575110 & 16.045981 \\
\hline S & 17.136932 & 11.059946 & 13.210886 \\
\hline S & 5.108548 & 11.128162 & 11.144998 \\
\hline S & 5.256349 & 17.195271 & 14.255142 \\
\hline S & 15.178976 & 10.891521 & 8.739050 \\
\hline S & 11.226490 & 4.962123 & 9.208303 \\
\hline S & 6.718729 & 8.854196 & 7.609774 \\
\hline S & 14.560460 & 5.236804 & 12.464682 \\
\hline S & 8.994723 & 16.529486 & 9.595551 \\
\hline S & 8.268816 & 17.819586 & 17.750291 \\
\hline S & 11.963592 & 7.322581 & 15.703379 \\
\hline S & 9.301672 & 12.307254 & 5.902335 \\
\hline S & 7.279197 & 8.437063 & 16.310621 \\
\hline S & 13.035411 & 13.711651 & 20.046249 \\
\hline C & 15.829127 & 15.250620 & 15.468440 \\
\hline $\mathrm{C}$ & 16.817606 & 10.215500 & 14.788914 \\
\hline C & 3.751129 & 10.125160 & 11.852814 \\
\hline $\mathrm{C}$ & 3.655957 & 16.879053 & 15.087935 \\
\hline C & 16.887310 & 10.441823 & 9.194874 \\
\hline $\mathrm{C}$ & 9.896777 & 3.943239 & 9.945667 \\
\hline C & 7.052591 & 7.412419 & 6.523361 \\
\hline C & 15.831753 & 6.412597 & 11.868779 \\
\hline C & 10.452716 & 17.576347 & 9.229893 \\
\hline C & 9.368890 & 19.194028 & 17.232147 \\
\hline $\mathrm{C}$ & 10.799693 & 5.984882 & 16.157420 \\
\hline $\mathrm{C}$ & 8.307214 & 13.841677 & 5.810685 \\
\hline $\mathrm{C}$ & 5.490693 & 8.838156 & 16.354736 \\
\hline $\mathrm{C}$ & 13.308633 & 15.267605 & 20.976926 \\
\hline $\mathrm{H}$ & 16.201296 & 15.143612 & 14.441596 \\
\hline $\mathrm{H}$ & 16.631481 & 15.631345 & 16.112249 \\
\hline $\mathrm{H}$ & 14.971347 & 15.930082 & 15.487103 \\
\hline $\mathrm{H}$ & 16.474156 & 10.922058 & 15.554555 \\
\hline $\mathrm{H}$ & 17.780113 & 9.787317 & 15.105553 \\
\hline $\mathrm{H}$ & 16.088413 & 9.404245 & 14.671621 \\
\hline $\mathrm{H}$ & 3.990525 & 9.773720 & 12.860855 \\
\hline $\mathrm{H}$ & 3.579315 & 9.269936 & 11.187723 \\
\hline $\mathrm{H}$ & 2.853503 & 10.755762 & 11.882265 \\
\hline $\mathrm{H}$ & 2.951880 & 16.524625 & 14.324989 \\
\hline $\mathrm{H}$ & 3.293867 & 17.823380 & 15.512490 \\
\hline $\mathrm{H}$ & 3.753803 & 16.127760 & 15.877145 \\
\hline $\mathrm{H}$ & 17.575110 & 11.057186 & 8.600864 \\
\hline $\mathrm{H}$ & 17.038750 & 9.383801 & 8.944119 \\
\hline $\mathrm{H}$ & 17.080064 & 10.594338 & 10.263843 \\
\hline $\mathrm{H}$ & 9.033359 & 3.985667 & 9.270711 \\
\hline $\mathrm{H}$ & 9.609157 & 4.305640 & 10.937023 \\
\hline $\mathrm{H}$ & 10.259860 & 2.910636 & 10.015199 \\
\hline $\mathrm{H}$ & 8.106933 & 7.358236 & 6.236628 \\
\hline $\mathrm{H}$ & 6.426938 & 7.509438 & 5.627757 \\
\hline $\mathrm{H}$ & 6.770427 & 6.505993 & 7.073107 \\
\hline $\mathrm{H}$ & 16.550019 & 6.567386 & 12.682651 \\
\hline $\mathrm{H}$ & 15.389127 & 7.370498 & 11.574050 \\
\hline $\mathrm{H}$ & 16.336015 & 5.948958 & 11.012698 \\
\hline $\mathrm{H}$ & 11.277196 & 17.365309 & 9.918525 \\
\hline $\mathrm{H}$ & 10.144897 & 18.624521 & 9.334136 \\
\hline $\mathrm{H}$ & 10.769338 & 17.379930 & 8.199017 \\
\hline $\mathrm{H}$ & 10.185360 & 19.266549 & 17.960346 \\
\hline $\mathrm{H}$ & 8.777067 & 20.117225 & 17.244935 \\
\hline $\mathrm{H}$ & 9.771465 & 19.021002 & 16.229417 \\
\hline $\mathrm{H}$ & 11.374602 & 5.204347 & 16.671489 \\
\hline $\mathrm{H}$ & 10.300777 & 5.564647 & 15.278896 \\
\hline $\mathrm{H}$ & 10.053289 & 6.408227 & 16.840442 \\
\hline $\mathrm{H}$ & 7.674699 & 13.784335 & 4.916958 \\
\hline $\mathrm{H}$ & 9.005744 & 14.682449 & 5.717612 \\
\hline
\end{tabular}




\begin{tabular}{|c|c|c|c|}
\hline $\mathrm{H}$ & 7.687364 & 13.977231 & 6.701835 \\
\hline $\mathrm{H}$ & 5.225648 & 9.066579 & 17.394644 \\
\hline $\mathrm{H}$ & 4.929462 & 7.958174 & 16.018068 \\
\hline $\mathrm{H}$ & 5.262066 & 9.701845 & 15.721146 \\
\hline $\mathrm{H}$ & 14.251342 & 15.170291 & 21.529434 \\
\hline $\mathrm{H}$ & 12.477786 & 15.380768 & 21.683375 \\
\hline $\mathrm{H}$ & 13.355464 & 16.138057 & 20.316000 \\
\hline $\mathrm{Au}$ & 11.287683 & 14.496493 & 15.596198 \\
\hline $\mathrm{Au}$ & 11.168187 & 12.877072 & 13.033731 \\
\hline $\mathrm{Au}$ & 8.521984 & 13.750026 & 12.749769 \\
\hline $\mathrm{Au}$ & 12.354813 & 8.167027 & 11.853753 \\
\hline $\mathrm{Au}$ & 9.355299 & 13.026981 & 16.982399 \\
\hline $\mathrm{Au}$ & 9.378184 & 11.806465 & 10.913725 \\
\hline $\mathrm{Au}$ & 11.346153 & 15.612856 & 13.110648 \\
\hline $\mathrm{Au}$ & 7.323509 & 12.846676 & 15.161762 \\
\hline $\mathrm{Au}$ & 11.165138 & 11.462245 & 15.485243 \\
\hline $\mathrm{Au}$ & 13.426185 & 14.231039 & 11.925855 \\
\hline $\mathrm{Au}$ & 12.781326 & 10.557998 & 13.211767 \\
\hline $\mathrm{Au}$ & 9.096170 & 11.056112 & 13.565686 \\
\hline $\mathrm{Au}$ & 9.466522 & 8.664873 & 11.761030 \\
\hline $\mathrm{Au}$ & 11.131998 & 9.910622 & 9.815753 \\
\hline $\mathrm{Au}$ & 9.840829 & 14.355336 & 9.936230 \\
\hline $\mathrm{Au}$ & 13.229868 & 6.406451 & 13.983261 \\
\hline $\mathrm{Au}$ & 9.542044 & 15.859667 & 17.332933 \\
\hline $\mathrm{Au}$ & 14.710044 & 12.926717 & 9.955766 \\
\hline $\mathrm{Au}$ & 6.230156 & 15.040205 & 13.956957 \\
\hline $\mathrm{Au}$ & 10.698958 & 7.222878 & 9.662345 \\
\hline $\mathrm{Au}$ & 8.328294 & 10.614357 & 16.421890 \\
\hline $\mathrm{Au}$ & 10.100463 & 12.100224 & 8.168840 \\
\hline $\mathrm{Au}$ & 13.482880 & 12.942445 & 14.680178 \\
\hline $\mathrm{Au}$ & 5.898587 & 12.577059 & 12.833860 \\
\hline $\mathrm{Au}$ & 8.932040 & 16.045528 & 14.587673 \\
\hline $\mathrm{Au}$ & 11.995045 & 12.585722 & 10.116431 \\
\hline $\mathrm{Au}$ & 11.473150 & 14.201427 & 18.315368 \\
\hline $\mathrm{Au}$ & 8.623463 & 9.021878 & 9.063692 \\
\hline $\mathrm{Au}$ & 7.913726 & 8.530610 & 14.009720 \\
\hline $\mathrm{Au}$ & 13.838936 & 10.161027 & 10.652466 \\
\hline $\mathrm{Au}$ & 14.326916 & 13.778393 & 18.088309 \\
\hline $\mathrm{Au}$ & 7.095175 & 9.963774 & 11.691356 \\
\hline $\mathrm{Au}$ & 6.804371 & 17.502865 & 15.974061 \\
\hline $\mathrm{Au}$ & 10.742776 & 8.884441 & 14.359588 \\
\hline $\mathrm{Au}$ & 15.228493 & 12.144457 & 12.541259 \\
\hline $\mathrm{Au}$ & 7.900783 & 10.615667 & 6.647065 \\
\hline $\mathrm{Au}$ & 8.903880 & 16.449420 & 11.970090 \\
\hline $\mathrm{Au}$ & 12.853478 & 5.244196 & 10.874141 \\
\hline \multicolumn{4}{|c|}{ \#figure $5 a$} \\
\hline S & 15.847544 & 14.079491 & 14.375349 \\
\hline $\mathrm{S}$ & 15.701532 & 10.009798 & 10.575069 \\
\hline $\mathrm{S}$ & 4.302088 & 13.588784 & 14.102035 \\
\hline S & 8.908910 & 17.835844 & 14.280469 \\
\hline $\mathrm{S}$ & 11.806595 & 10.738515 & 6.788114 \\
\hline S & 7.619437 & 6.724087 & 10.987399 \\
\hline $\mathrm{S}$ & 4.119894 & 10.560287 & 11.544289 \\
\hline $\mathrm{S}$ & 11.604439 & 6.432781 & 11.080623 \\
\hline S & 12.189445 & 17.473334 & 11.155226 \\
\hline S & 11.606926 & 13.394043 & 18.740251 \\
\hline $\mathrm{S}$ & 11.682436 & 6.473285 & 15.149464 \\
\hline $\mathrm{S}$ & 8.913741 & 13.408072 & 6.779643 \\
\hline $\mathrm{S}$ & 8.889373 & 10.324870 & 18.653749 \\
\hline S & 15.892181 & 10.153428 & 14.625970 \\
\hline C & 15.451946 & 15.624120 & 15.265499 \\
\hline C & 17.381118 & 10.479600 & 11.124228 \\
\hline $\mathrm{C}$ & 4.026842 & 14.952656 & 15.286587 \\
\hline $\mathrm{C}$ & 7.557811 & 18.083184 & 15.486275 \\
\hline C & 13.170682 & 9.550114 & 6.524604 \\
\hline C & 8.134553 & 5.018044 & 11.406993 \\
\hline C & 3.990039 & 9.161724 & 10.369332 \\
\hline $\mathrm{C}$ & 12.908828 & 6.520709 & 9.795112 \\
\hline $\mathrm{C}$ & 11.517469 & 19.013415 & 11.873612 \\
\hline C & 12.766164 & 14.790723 & 18.948104 \\
\hline
\end{tabular}




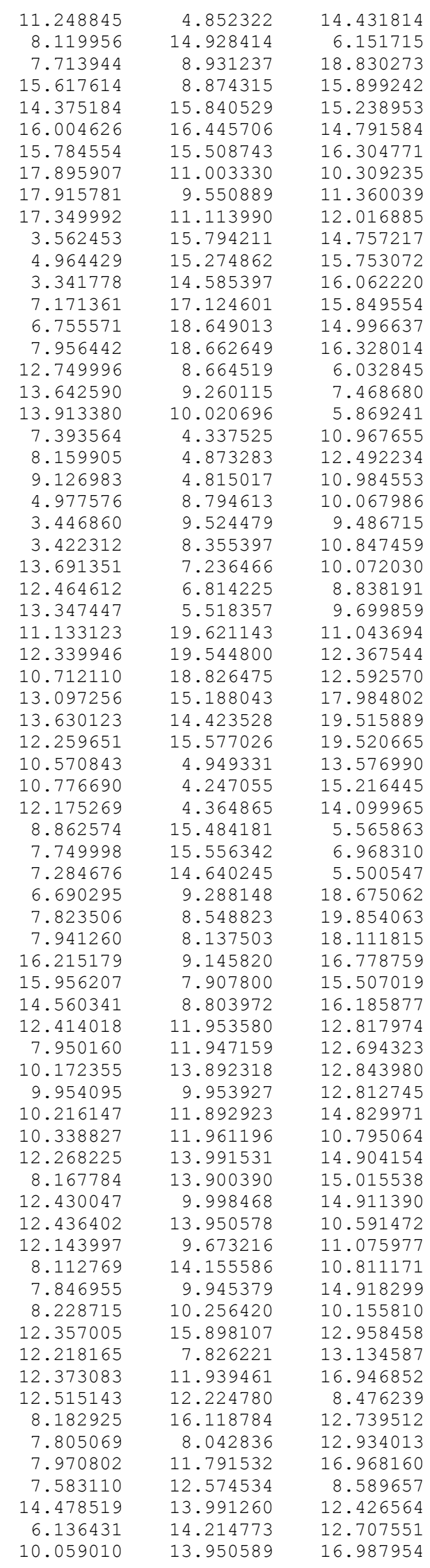




\begin{tabular}{|c|c|c|c|}
\hline $\mathrm{Au}$ & 10.221177 & 13.963659 & 8.767573 \\
\hline $\mathrm{Au}$ & 14.323220 & 9.777320 & 12.777023 \\
\hline $\mathrm{Au}$ & 5.761893 & 10.023800 & 13.142735 \\
\hline $\mathrm{Au}$ & 10.412161 & 9.909416 & 16.845337 \\
\hline $\mathrm{Au}$ & 10.643471 & 9.880386 & 8.703207 \\
\hline $\mathrm{Au}$ & 14.437671 & 12.149105 & 15.034414 \\
\hline $\mathrm{Au}$ & 6.002726 & 12.094979 & 15.051065 \\
\hline $\mathrm{Au}$ & 10.281377 & 15.933156 & 14.930700 \\
\hline $\mathrm{Au}$ & 9.812607 & 7.969958 & 14.872700 \\
\hline $\mathrm{Au}$ & 14.391191 & 11.976423 & 10.533036 \\
\hline $\mathrm{Au}$ & 5.970557 & 11.793258 & 10.546995 \\
\hline $\mathrm{Au}$ & 10.361011 & 15.910223 & 10.730908 \\
\hline $\mathrm{Au}$ & 9.761456 & 8.030063 & 10.528045 \\
\hline \multicolumn{4}{|c|}{ \#figure 5b } \\
\hline S & 14.606002 & 13.265536 & 17.070863 \\
\hline S & 9.964910 & 13.796476 & 17.160544 \\
\hline S & 14.637144 & 8.969828 & 17.011082 \\
\hline S & 10.047165 & 9.167127 & 17.907227 \\
\hline S & 14.946568 & 13.482392 & 7.624943 \\
\hline S & 10.613142 & 13.834426 & 7.616720 \\
\hline S & 14.568990 & 8.794551 & 7.603815 \\
\hline S & 9.895139 & 9.214476 & 7.828584 \\
\hline S & 14.902575 & 16.739085 & 14.670389 \\
\hline S & 15.226824 & 16.820038 & 9.982876 \\
\hline S & 10.569338 & 17.014150 & 14.381087 \\
\hline S & 10.534897 & 16.784275 & 10.011368 \\
\hline S & 15.447232 & 6.290258 & 14.269863 \\
\hline S & 14.609389 & 5.780979 & 9.980269 \\
\hline S & 10.876188 & 6.802973 & 14.997284 \\
\hline S & 9.963697 & 5.907369 & 9.456981 \\
\hline S & 17.390708 & 13.962092 & 14.860674 \\
\hline S & 18.012625 & 13.598892 & 10.538748 \\
\hline S & 18.043199 & 9.320983 & 15.044391 \\
\hline S & 18.508219 & 9.237603 & 10.392059 \\
\hline S & 7.057530 & 13.099103 & 14.223159 \\
\hline S & 7.855779 & 13.726517 & 10.144190 \\
\hline S & 7.914929 & 9.217283 & 14.754694 \\
\hline S & 6.646073 & 9.210940 & 10.217981 \\
\hline C & 14.729384 & 12.743806 & 18.826241 \\
\hline C & 9.906559 & 14.555116 & 18.834359 \\
\hline C & 15.446363 & 9.202600 & 18.643256 \\
\hline C & 10.004893 & 8.954264 & 19.727302 \\
\hline C & 14.600848 & 13.828015 & 5.856975 \\
\hline C & 10.983221 & 14.037443 & 5.833032 \\
\hline C & 14.910702 & 8.477795 & 5.823718 \\
\hline C & 9.277992 & 8.804674 & 6.155712 \\
\hline C & 14.565067 & 18.474864 & 15.147448 \\
\hline $\mathrm{C}$ & 15.773605 & 18.511001 & 9.544400 \\
\hline $\mathrm{C}$ & 10.997696 & 18.757369 & 14.028821 \\
\hline C & 10.060223 & 18.510457 & 10.389923 \\
\hline $\mathrm{C}$ & 15.666469 & 4.478057 & 14.113378 \\
\hline $\mathrm{C}$ & 14.946079 & 4.037130 & 10.422920 \\
\hline $\mathrm{C}$ & 10.217651 & 5.440162 & 13.968896 \\
\hline C & 9.370978 & 4.379072 & 10.270314 \\
\hline $\mathrm{C}$ & 19.039858 & 14.721117 & 14.642968 \\
\hline C & 19.730475 & 13.484825 & 11.155749 \\
\hline $\mathrm{C}$ & 19.756695 & 9.046470 & 15.630027 \\
\hline C & 20.249199 & 9.736609 & 10.089649 \\
\hline C & 5.624763 & 12.239408 & 13.486800 \\
\hline C & 6.337509 & 14.705224 & 10.448681 \\
\hline $\mathrm{C}$ & 6.341656 & 9.549699 & 15.631308 \\
\hline $\mathrm{C}$ & 4.818939 & 9.329602 & 10.356035 \\
\hline $\mathrm{H}$ & 14.574988 & 13.630272 & 19.454301 \\
\hline $\mathrm{H}$ & 15.739859 & 12.349013 & 18.991409 \\
\hline $\mathrm{H}$ & 13.981856 & 11.982088 & 19.073937 \\
\hline $\mathrm{H}$ & 10.592883 & 14.064258 & 19.530604 \\
\hline $\mathrm{H}$ & 10.174524 & 15.613380 & 18.726416 \\
\hline $\mathrm{H}$ & 8.875865 & 14.472872 & 19.201998 \\
\hline $\mathrm{H}$ & 14.952871 & 9.962349 & 19.254918 \\
\hline $\mathrm{H}$ & 16.488428 & 9.493476 & 18.458300 \\
\hline
\end{tabular}




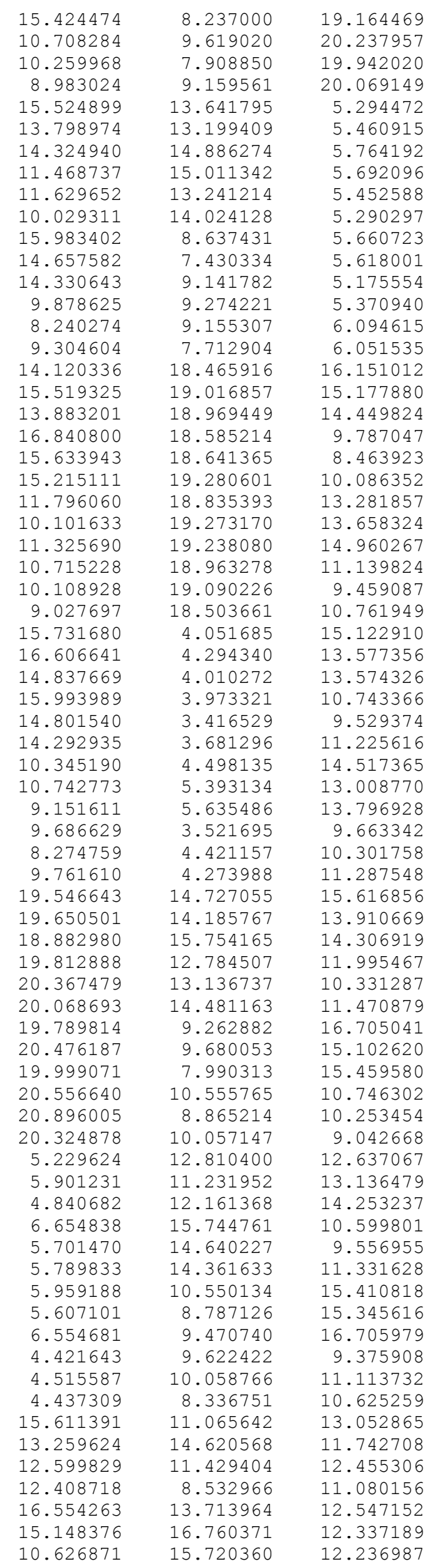




\begin{tabular}{|c|c|c|c|}
\hline $\mathrm{Au}$ & 8.677312 & 13.272853 & 12.390055 \\
\hline $\mathrm{Au}$ & 7.442341 & 9.149772 & 12.447415 \\
\hline $\mathrm{Au}$ & 9.733654 & 7.791590 & 10.953298 \\
\hline $\mathrm{Au}$ & 14.624253 & 7.111610 & 12.084862 \\
\hline $\mathrm{Au}$ & 18.233533 & 9.265143 & 12.715209 \\
\hline $\mathrm{Au}$ & 11.704114 & 10.876717 & 15.074851 \\
\hline $\mathrm{Au}$ & 17.722264 & 11.646402 & 14.986086 \\
\hline $\mathrm{Au}$ & 14.068400 & 13.272697 & 14.034686 \\
\hline $\mathrm{Au}$ & 12.686511 & 15.686896 & 14.367933 \\
\hline $\mathrm{Au}$ & 11.011095 & 13.442417 & 13.968339 \\
\hline $\mathrm{Au}$ & 9.025699 & 11.408994 & 14.466127 \\
\hline $\mathrm{Au}$ & 10.721969 & 8.658486 & 13.443126 \\
\hline $\mathrm{Au}$ & 13.170293 & 6.549688 & 14.736597 \\
\hline $\mathrm{Au}$ & 13.661801 & 9.186043 & 13.720702 \\
\hline $\mathrm{Au}$ & 14.503411 & 11.110947 & 15.743617 \\
\hline $\mathrm{Au}$ & 12.292319 & 13.558765 & 16.792286 \\
\hline $\mathrm{Au}$ & 9.974248 & 11.473984 & 17.503133 \\
\hline $\mathrm{Au}$ & 12.342374 & 9.106060 & 17.411756 \\
\hline $\mathrm{Au}$ & 12.384844 & 11.010568 & 9.713267 \\
\hline $\mathrm{Au}$ & 17.126610 & 11.257742 & 10.550077 \\
\hline $\mathrm{Au}$ & 14.695471 & 12.517942 & 10.692085 \\
\hline $\mathrm{Au}$ & 12.872912 & 16.796489 & 9.921346 \\
\hline $\mathrm{Au}$ & 10.968973 & 13.201797 & 10.919701 \\
\hline $\mathrm{Au}$ & 7.308153 & 11.460471 & 10.153655 \\
\hline $\mathrm{Au}$ & 10.045491 & 10.501609 & 11.523657 \\
\hline $\mathrm{Au}$ & 12.288504 & 5.888151 & 9.739877 \\
\hline $\mathrm{Au}$ & 14.919417 & 9.604799 & 10.761219 \\
\hline $\mathrm{Au}$ & 14.772489 & 11.139850 & 7.808025 \\
\hline $\mathrm{Au}$ & 12.808541 & 13.680370 & 8.876711 \\
\hline $\mathrm{Au}$ & 10.226952 & 11.537119 & 7.809803 \\
\hline $\mathrm{Au}$ & 12.226558 & 8.897122 & 7.757269 \\
\hline \multicolumn{4}{|c|}{ \#figure $5 \mathrm{c}$} \\
\hline S & 8.187967 & 5.539307 & 13.385649 \\
\hline S & 5.586440 & 7.703703 & 10.179429 \\
\hline S & 5.904660 & 8.744639 & 17.504330 \\
\hline S & 4.166870 & 10.796790 & 13.717808 \\
\hline S & 12.638685 & 6.167266 & 14.507689 \\
\hline S & 10.874095 & 7.533775 & 10.414854 \\
\hline $\mathrm{S}$ & 11.912812 & 12.278972 & 6.906942 \\
\hline S & 8.721232 & 15.584904 & 6.341060 \\
\hline $\mathrm{S}$ & 15.879545 & 12.844853 & 14.091947 \\
\hline S & 14.457296 & 12.708901 & 18.542329 \\
\hline S & 14.471274 & 16.748531 & 11.439700 \\
\hline $\mathrm{S}$ & 13.232615 & 16.980516 & 15.896378 \\
\hline S & 10.357375 & 18.401743 & 13.431682 \\
\hline S & 8.875835 & 15.965614 & 17.108675 \\
\hline S & 5.212055 & 15.370016 & 14.789280 \\
\hline S & 3.918593 & 13.927148 & 10.531518 \\
\hline S & 15.571527 & 9.421287 & 10.701487 \\
\hline S & 14.109785 & 13.585631 & 9.291363 \\
\hline S & 7.867790 & 12.858617 & 18.392784 \\
\hline S & 10.023325 & 8.594047 & 17.525587 \\
\hline S & 8.882131 & 8.504806 & 7.350099 \\
\hline S & 6.289259 & 12.360710 & 7.396593 \\
\hline S & 7.443394 & 16.383494 & 10.716811 \\
\hline S & 13.101198 & 10.068720 & 14.552533 \\
\hline C & 7.017436 & 5.172108 & 14.744067 \\
\hline C & 5.981650 & 6.766885 & 8.661062 \\
\hline C & 4.447557 & 8.882058 & 18.614651 \\
\hline $\mathrm{C}$ & 3.521789 & 9.379212 & 12.758574 \\
\hline C & 12.204544 & 4.392903 & 14.555992 \\
\hline $\mathrm{C}$ & 12.233776 & 7.416259 & 9.196439 \\
\hline C & 11.344676 & 10.939772 & 5.800157 \\
\hline $\mathrm{C}$ & 9.626578 & 17.083551 & 5.800760 \\
\hline C & 16.722407 & 14.443895 & 13.835585 \\
\hline C & 14.819035 & 11.038309 & 19.198725 \\
\hline C & 14.012190 & 18.314436 & 10.621770 \\
\hline C & 14.730202 & 16.528711 & 16.848871 \\
\hline $\mathrm{C}$ & 8.848337 & 19.103051 & 12.679772 \\
\hline $\mathrm{C}$ & 9.911668 & 16.669838 & 18.448172 \\
\hline
\end{tabular}




$$
\begin{aligned}
& 4.228457 \\
& 2.546430 \\
& 17.278183 \\
& 15.631553 \\
& 8.843443 \\
& 10.857040 \\
& 8.517009 \\
& 4.656707 \\
& 5.752459 \\
& 13.967708 \\
& 6.479597 \\
& 7.600051 \\
& 6.307113 \\
& 7.013694 \\
& 5.283027 \\
& 5.831262 \\
& 3.998505 \\
& 4.786389 \\
& 3.714053 \\
& 4.303542 \\
& 3.143099 \\
& 2.702383 \\
& 11.137577 \\
& 12.470429 \\
& 12.800550 \\
& 13.116044 \\
& 11.839712 \\
& 12.492226 \\
& 10.323675 \\
& 12.025650 \\
& 11.413201 \\
& 10.482868 \\
& 8.927634 \\
& 9.971013 \\
& 16.061139 \\
& 17.021920 \\
& 17.614328 \\
& 14.501748 \\
& 14.284621 \\
& 15.899009 \\
& 12.974638 \\
& 14.140708 \\
& 14.687536 \\
& 15.150266 \\
& 14.443265 \\
& 15.467487 \\
& 8.180737 \\
& 9.159692 \\
& 8.346207 \\
& 10.976244 \\
& 9.724190 \\
& 9.611651 \\
& 3.206732 \\
& 4.684855 \\
& 4.218552 \\
& 2.773395 \\
& 2.376899 \\
& 1.646502 \\
& 17.935360 \\
& 17.582200 \\
& 17.331654 \\
& 16.211732 \\
& 15.335462 \\
& 16.235324 \\
& 8.180654 \\
& 9.281100 \\
& 9.652252 \\
& 10.282764 \\
& 11.890346 \\
& 10.838776
\end{aligned}
$$




\begin{tabular}{|c|c|c|c|}
\hline $\mathrm{H}$ & 9.125858 & 6.903652 & 5.589047 \\
\hline $\mathrm{H}$ & 8.784441 & 8.511224 & 4.893494 \\
\hline $\mathrm{H}$ & 4.768872 & 11.744869 & 5.631209 \\
\hline $\mathrm{H}$ & 3.966369 & 12.737174 & 6.889548 \\
\hline $\mathrm{H}$ & 4.267240 & 10.991086 & 7.176458 \\
\hline $\mathrm{H}$ & 5.203013 & 17.057011 & 11.287680 \\
\hline $\mathrm{H}$ & 5.219396 & 16.299102 & 9.668050 \\
\hline $\mathrm{H}$ & 5.842474 & 17.969295 & 9.876682 \\
\hline $\mathrm{H}$ & 14.298916 & 8.167871 & 15.559751 \\
\hline $\mathrm{H}$ & 13.289485 & 9.063355 & 16.744911 \\
\hline $\mathrm{H}$ & 14.832995 & 9.778912 & 16.162757 \\
\hline $\mathrm{Au}$ & 11.281600 & 12.383252 & 11.864849 \\
\hline $\mathrm{Au}$ & 6.054286 & 11.716998 & 12.458645 \\
\hline $\mathrm{Au}$ & 9.824727 & 14.595042 & 13.494463 \\
\hline $\mathrm{Au}$ & 8.066070 & 9.911351 & 15.220662 \\
\hline $\mathrm{Au}$ & 10.641567 & 9.956580 & 13.806779 \\
\hline $\mathrm{Au}$ & 8.609172 & 11.800516 & 10.617181 \\
\hline $\mathrm{Au}$ & 11.466296 & 12.556613 & 14.699022 \\
\hline $\mathrm{Au}$ & 7.285627 & 14.240702 & 14.566709 \\
\hline $\mathrm{Au}$ & 11.247732 & 7.312593 & 16.003634 \\
\hline $\mathrm{Au}$ & 12.823541 & 14.810850 & 10.976646 \\
\hline $\mathrm{Au}$ & 10.643521 & 9.856108 & 10.843351 \\
\hline $\mathrm{Au}$ & 8.252542 & 15.900880 & 8.596999 \\
\hline $\mathrm{Au}$ & 7.059726 & 10.767353 & 17.836290 \\
\hline $\mathrm{Au}$ & 6.660246 & 9.840151 & 10.077707 \\
\hline $\mathrm{Au}$ & 13.818599 & 16.912516 & 13.649867 \\
\hline $\mathrm{Au}$ & 11.767032 & 6.965538 & 12.484550 \\
\hline $\mathrm{Au}$ & 12.381004 & 12.670596 & 17.361772 \\
\hline $\mathrm{Au}$ & 10.834159 & 12.014732 & 9.030984 \\
\hline $\mathrm{Au}$ & 7.407376 & 14.161392 & 11.724310 \\
\hline $\mathrm{Au}$ & 6.962965 & 6.779612 & 11.813173 \\
\hline $\mathrm{Au}$ & 9.715779 & 13.589306 & 16.782730 \\
\hline $\mathrm{Au}$ & 13.708872 & 12.881240 & 13.032939 \\
\hline $\mathrm{Au}$ & 4.558582 & 14.475703 & 12.714020 \\
\hline $\mathrm{Au}$ & 11.882949 & 15.051384 & 15.809320 \\
\hline $\mathrm{Au}$ & 10.085191 & 14.487044 & 10.450398 \\
\hline $\mathrm{Au}$ & 14.270942 & 9.668283 & 12.607920 \\
\hline $\mathrm{Au}$ & 5.185375 & 9.776808 & 15.528160 \\
\hline $\mathrm{Au}$ & 10.268460 & 10.900204 & 16.812262 \\
\hline $\mathrm{Au}$ & 7.558765 & 10.401102 & 7.435498 \\
\hline $\mathrm{Au}$ & 15.221387 & 12.790752 & 16.318860 \\
\hline $\mathrm{Au}$ & 8.703930 & 12.094812 & 13.570345 \\
\hline $\mathrm{Au}$ & 9.621472 & 17.044453 & 15.179431 \\
\hline $\mathrm{Au}$ & 9.223844 & 7.610479 & 13.970290 \\
\hline $\mathrm{Au}$ & 14.840446 & 11.566859 & 10.126314 \\
\hline $\mathrm{Au}$ & 5.935090 & 12.557582 & 9.807504 \\
\hline $\mathrm{Au}$ & 11.171488 & 16.543471 & 12.160435 \\
\hline $\mathrm{Au}$ & 7.990708 & 9.560920 & 12.462888 \\
\hline $\mathrm{Au}$ & 10.329810 & 13.947382 & 6.638015 \\
\hline \multicolumn{4}{|c|}{ \#figure $6 a$} \\
\hline $\mathrm{S}$ & 8.690570 & 5.982718 & 14.078974 \\
\hline $\mathrm{S}$ & 5.926130 & 7.582983 & 10.657213 \\
\hline $\mathrm{S}$ & 5.379879 & 9.103895 & 16.984722 \\
\hline $\mathrm{S}$ & 3.389162 & 11.061961 & 13.219561 \\
\hline S & 14.049978 & 7.546650 & 15.034195 \\
\hline S & 12.682919 & 7.503788 & 10.557638 \\
\hline $\mathrm{S}$ & 10.753420 & 12.681912 & 6.643937 \\
\hline $\mathrm{S}$ & 6.813065 & 14.923301 & 7.725810 \\
\hline $\mathrm{S}$ & 16.036024 & 12.782228 & 14.525370 \\
\hline S & 13.686228 & 11.831506 & 18.466034 \\
\hline $\mathrm{S}$ & 14.510689 & 16.034310 & 11.411699 \\
\hline $\mathrm{S}$ & 12.824791 & 16.376208 & 15.765875 \\
\hline $\mathrm{C}$ & 7.587551 & 5.447883 & 15.440814 \\
\hline $\mathrm{C}$ & 6.447131 & 6.603299 & 9.203035 \\
\hline C & 4.218052 & 9.582216 & 18.315829 \\
\hline C & 2.671506 & 9.679878 & 12.260755 \\
\hline C & 13.439616 & 5.946219 & 15.680704 \\
\hline $\mathrm{C}$ & 14.201882 & 7.595527 & 9.549329 \\
\hline $\mathrm{C}$ & 10.271473 & 10.985009 & 6.163470 \\
\hline C & 7.228319 & 16.567575 & 7.033385 \\
\hline
\end{tabular}




\begin{tabular}{|c|c|c|}
\hline 16.929305 & 14.352201 & 14.781253 \\
\hline 13.856736 & 10.024114 & 18.680310 \\
\hline 13.947662 & 17.602853 & 10.645651 \\
\hline 14.200039 & 16.005321 & 16.910307 \\
\hline 6.882047 & 6.234039 & 15.727726 \\
\hline 8.225286 & 5.194167 & 16.296884 \\
\hline 7.045279 & 4.555740 & 15.105136 \\
\hline 7.519691 & 6.713759 & 9.015863 \\
\hline 5.878581 & 6.967816 & 8.338793 \\
\hline 6.204782 & 5.550051 & 9.390673 \\
\hline 4.074147 & 10.666891 & 18.354897 \\
\hline 4.646461 & 9.234684 & 19.264657 \\
\hline 3.257344 & 9.082925 & 18.141967 \\
\hline 3.399211 & 8.882336 & 12.078986 \\
\hline 2.328682 & 10.090046 & 11.302735 \\
\hline 1.814501 & 9.284780 & 12.820408 \\
\hline 12.407751 & 5.755499 & 15.367668 \\
\hline 13.486300 & 5.993640 & 16.776338 \\
\hline 14.095231 & 5.144967 & 15.318578 \\
\hline 14.820058 & 8.453243 & 9.835597 \\
\hline 13.905290 & 7.688773 & 8.497283 \\
\hline 14.764959 & 6.665107 & 9.692308 \\
\hline 9.725827 & 10.485392 & 6.973307 \\
\hline 11.191082 & 10.430382 & 5.939348 \\
\hline 9.645660 & 11.045811 & 5.264554 \\
\hline 8.170404 & 16.945985 & 7.442133 \\
\hline 6.409908 & 17.252952 & 7.285133 \\
\hline 7.306411 & 16.465696 & 5.944086 \\
\hline 16.248782 & 15.176750 & 15.018242 \\
\hline 17.464740 & 14.577215 & 13.850731 \\
\hline 17.649967 & 14.207656 & 15.596360 \\
\hline 13.493829 & 9.483005 & 17.798983 \\
\hline 13.267475 & 9.740966 & 19.561289 \\
\hline 14.915699 & 9.794577 & 18.853579 \\
\hline 12.858785 & 17.708489 & 10.713756 \\
\hline 14.251812 & 17.578642 & 9.592197 \\
\hline 14.441137 & 18.437030 & 11.158601 \\
\hline 14.525068 & 14.958384 & 16.821200 \\
\hline 13.835195 & 16.184348 & 17.929849 \\
\hline 15.033264 & 16.687038 & 16.698123 \\
\hline 11.065465 & 12.396671 & 11.640607 \\
\hline 5.574765 & 11.446235 & 12.341189 \\
\hline 10.045852 & 13.646329 & 13.837471 \\
\hline 7. 328812 & 9.800341 & 13.967240 \\
\hline 10.350743 & 10.703667 & 13.822987 \\
\hline 8.361955 & 11.936123 & 12.349940 \\
\hline 12.360383 & 12.252163 & 14.955871 \\
\hline 7.770602 & 14.880935 & 15.016993 \\
\hline 12.143468 & 9.025336 & 15.222824 \\
\hline 12.856918 & 14.404825 & 10.707198 \\
\hline 11.550052 & 9.640990 & 10.529005 \\
\hline 7.202261 & 15.233000 & 10.064885 \\
\hline 7.262826 & 10.654502 & 16.904655 \\
\hline 6.785580 & 9.767201 & 10.293975 \\
\hline 13.624018 & 16.053118 & 13.587325 \\
\hline 13.411278 & 7.578216 & 12.768885 \\
\hline 11.493386 & 12.285438 & 17.639874 \\
\hline 11.509838 & 12.684541 & 8.905124 \\
\hline 8.412653 & 15.986120 & 12.329476 \\
\hline 7.330697 & 6.899650 & 12.402213 \\
\hline 9.040560 & 12.814864 & 16.548917 \\
\hline 14.248956 & 12.978556 & 12.950055 \\
\hline 6.494482 & 14.107660 & 12.554740 \\
\hline 11.131506 & 14.652715 & 16.243373 \\
\hline 10.011285 & 14.817443 & 10.013893 \\
\hline 13.095157 & 10.414067 & 12.940423 \\
\hline 4.513105 & 10.096608 & 15.047906 \\
\hline 9.954158 & 10.158737 & 16.509744 \\
\hline 9.289734 & 11.000852 & 9.754240 \\
\hline 14.781446 & 12.330914 & 16.451179 \\
\hline 6.719254 & 12.421950 & 14.872311 \\
\hline
\end{tabular}




\begin{tabular}{|c|c|c|c|}
\hline $\mathrm{Au}$ & 9.858946 & 16.597668 & 14.600573 \\
\hline $\mathrm{Au}$ & 9.517018 & 8.124862 & 14.681137 \\
\hline $\mathrm{Au}$ & 13.601775 & 11.685678 & 10.523610 \\
\hline $\mathrm{Au}$ & 6.937001 & 12.465215 & 10.005731 \\
\hline $\mathrm{Au}$ & 11.126973 & 15.874269 & 12.277920 \\
\hline $\mathrm{Au}$ & 9.099933 & 9.106596 & 11.879367 \\
\hline $\mathrm{Au}$ & 8.805136 & 13.718157 & 7.466353 \\
\hline \multicolumn{4}{|c|}{ \#figure 6b } \\
\hline S & 7.502422 & 4.554555 & 12.455475 \\
\hline $\mathrm{S}$ & 4.289453 & 7.095596 & 10.235609 \\
\hline S & 5.611445 & 14.322771 & 17.972670 \\
\hline $\mathrm{S}$ & 3.066304 & 11.740288 & 15.058779 \\
\hline $\mathrm{S}$ & 11.943868 & 6.218976 & 9.710129 \\
\hline S & 10.753579 & 9.400777 & 6.531604 \\
\hline S & 13.015315 & 16.076873 & 8.948893 \\
\hline $\mathrm{S}$ & 9.211284 & 14.607862 & 6.668242 \\
\hline $\mathrm{S}$ & 15.456792 & 9.940998 & 15.456010 \\
\hline $\mathrm{S}$ & 12.717153 & 7.894890 & 18.674082 \\
\hline S & 15.001698 & 16.068492 & 15.085549 \\
\hline S & 12.573869 & 15.117654 & 18.963169 \\
\hline C & 8.495073 & 3.654965 & 11.204932 \\
\hline C & 5.085284 & 7.593270 & 8.659240 \\
\hline $\mathrm{C}$ & 5.630901 & 16.031751 & 17.314253 \\
\hline C & 2.322421 & 10.587826 & 16.275105 \\
\hline C & 13.663800 & 6.522095 & 10.265445 \\
\hline $\mathrm{C}$ & 12.191528 & 10.013333 & 5.575623 \\
\hline $\mathrm{C}$ & 14.392335 & 15.008175 & 8.393238 \\
\hline $\mathrm{C}$ & 8.491961 & 16.154500 & 5.996639 \\
\hline C & 16.512846 & 11.148356 & 16.343727 \\
\hline C & 12.591501 & 6.155884 & 18.120859 \\
\hline $\mathrm{C}$ & 14.553690 & 17.806589 & 14.729614 \\
\hline $\mathrm{C}$ & 13.563759 & 13.843610 & 19.830960 \\
\hline $\mathrm{H}$ & 7.907537 & 2.797152 & 10.855924 \\
\hline $\mathrm{H}$ & 9.408194 & 3.299450 & 11.698387 \\
\hline $\mathrm{H}$ & 8.762998 & 4.295939 & 10.359766 \\
\hline $\mathrm{H}$ & 6.145785 & 7.827991 & 8.798096 \\
\hline $\mathrm{H}$ & 4.559385 & 8.479267 & 8.283476 \\
\hline $\mathrm{H}$ & 4.972795 & 6.766717 & 7.947093 \\
\hline $\mathrm{H}$ & 6.160206 & 16.660610 & 18.040047 \\
\hline $\mathrm{H}$ & 4.593678 & 16.374280 & 17.217006 \\
\hline $\mathrm{H}$ & 6.134117 & 16.077635 & 16.343082 \\
\hline $\mathrm{H}$ & 3.083335 & 10.097693 & 16.889398 \\
\hline $\mathrm{H}$ & 1.759113 & 9.834903 & 15.709693 \\
\hline $\mathrm{H}$ & 1.637142 & 11.162371 & 16.910096 \\
\hline $\mathrm{H}$ & 13.863457 & 7.589309 & 10.399820 \\
\hline $\mathrm{H}$ & 14.345504 & 6.105156 & 9.513986 \\
\hline $\mathrm{H}$ & 13.801105 & 5.996953 & 11.218539 \\
\hline $\mathrm{H}$ & 13.072970 & 10.157696 & 6.206870 \\
\hline $\mathrm{H}$ & 11.899294 & 10.968801 & 5.122614 \\
\hline $\mathrm{H}$ & 12.411486 & 9.284645 & 4.785629 \\
\hline $\mathrm{H}$ & 14.158472 & 13.949276 & 8.540402 \\
\hline $\mathrm{H}$ & 15.280781 & 15.278254 & 8.976794 \\
\hline $\mathrm{H}$ & 14.572355 & 15.209785 & 7.330033 \\
\hline $\mathrm{H}$ & 8.438458 & 16.938957 & 6.757561 \\
\hline $\mathrm{H}$ & 7.484193 & 15.917567 & 5.634751 \\
\hline $\mathrm{H}$ & 9.118220 & 16.486097 & 5.159463 \\
\hline $\mathrm{H}$ & 15.910845 & 11.855076 & 16.923858 \\
\hline $\mathrm{H}$ & 17.102072 & 11.688964 & 15.593821 \\
\hline $\mathrm{H}$ & 17.182709 & 10.590960 & 17.009874 \\
\hline $\mathrm{H}$ & 12.244976 & 6.083460 & 17.085201 \\
\hline $\mathrm{H}$ & 11.877889 & 5.652628 & 18.784592 \\
\hline $\mathrm{H}$ & 13.577155 & 5.685229 & 18.220326 \\
\hline $\mathrm{H}$ & 13.471295 & 17.928260 & 14.624830 \\
\hline $\mathrm{H}$ & 15.049894 & 18.091733 & 13.793872 \\
\hline $\mathrm{H}$ & 14.924625 & 18.433870 & 15.549277 \\
\hline $\mathrm{H}$ & 13.715970 & 12.959333 & 19.203239 \\
\hline $\mathrm{H}$ & 13.014860 & 13.561279 & 20.737527 \\
\hline $\mathrm{H}$ & 14.529346 & 14.284429 & 20.105868 \\
\hline $\mathrm{Au}$ & 10.902913 & 13.059382 & 9.879879 \\
\hline $\mathrm{Au}$ & 4.682801 & 10.457016 & 13.888285 \\
\hline
\end{tabular}




$\begin{array}{lrrr}\mathrm{Au} & 11.997045 & 10.915042 & 16.583963 \\ \mathrm{Au} & 7.050034 & 9.020306 & 13.889276 \\ \mathrm{Au} & 8.178044 & 11.612790 & 14.097779 \\ \mathrm{Au} & 8.429229 & 11.616517 & 9.969798 \\ \mathrm{Au} & 9.779078 & 9.070078 & 13.292518 \\ \mathrm{Au} & 9.523070 & 13.502644 & 15.690094 \\ \mathrm{Au} & 10.577684 & 7.720522 & 10.969925 \\ \mathrm{Au} & 13.734646 & 14.771064 & 13.525014 \\ \mathrm{Au} & 10.671471 & 10.732189 & 8.494136 \\ \mathrm{Au} & 8.274451 & 14.182402 & 8.818126 \\ \mathrm{Au} & 7.889202 & 13.660151 & 17.907666 \\ \mathrm{Au} & 5.216863 & 8.588788 & 11.873018 \\ \mathrm{Au} & 13.730983 & 15.511588 & 16.972714 \\ \mathrm{Au} & 11.496583 & 7.788342 & 8.041788 \\ \mathrm{Au} & 10.698175 & 9.018585 & 17.996414 \\ \mathrm{Au} & 12.351055 & 15.210953 & 11.084342 \\ \mathrm{Au} & 8.204302 & 14.468871 & 13.505206 \\ \mathrm{Au} & 5.875203 & 5.716948 & 11.260435 \\ \mathrm{Au} & 9.492927 & 11.466562 & 17.727756 \\ \mathrm{Au} & 13.757860 & 11.268788 & 14.428694 \\ \mathrm{Au} & 9.435550 & 15.215626 & 11.140773 \\ \mathrm{Au} & 10.656330 & 13.928872 & 18.193542 \\ \mathrm{Au} & 10.916366 & 14.594061 & 13.410561 \\ \mathrm{Au} & 10.827527 & 10.653677 & 11.293856 \\ \mathrm{Au} & 4.513983 & 13.050912 & 16.352352 \\ \mathrm{Au} & 8.969762 & 9.515352 & 15.890259 \\ \mathrm{Au} & 7.014843 & 13.707744 & 11.176157 \\ \mathrm{Au} & 14.049349 & 8.977662 & 17.069235 \\ \mathrm{Au} & 5.861847 & 13.139700 & 13.761379 \\ \mathrm{Au} & 12.340348 & 13.463651 & 15.599881 \\ \mathrm{Au} & 8.450983 & 6.751452 & 12.644342 \\ \mathrm{Au} & 12.627419 & 12.613555 & 12.202589 \\ \mathrm{Au} & 6.396611 & 11.091974 & 11.764555 \\ \mathrm{Au} & 10.944326 & 11.423870 & 14.032712 \\ \mathrm{Au} & 8.154430 & 9.087226 & 11.000651 \\ \mathrm{Au} & 11.116960 & 15.257874 & 7.882729\end{array}$

